\title{
ON QUINTIC SURFACES OF GENERAL TYPE
}

\author{
BY
}

JIN-GEN YANG

\begin{abstract}
The study of quintic surfaces is of special interest because 5 is the lowest degree of surfaces of general type. The aim of this paper is to give a classification of the quintic surfaces of general type over the complex number field $\mathbf{C}$.

We show that if $S$ is an irreducible quintic surface of general type then it must be normal, and it has only elliptic double or triple points as essential singularities. Then we classify all such surfaces in terms of the classification of the elliptic double and triple points. We give many examples in order to verify the existence of various types of quintic surfaces of general type. We also make a study of the double or triple covering of a quintic surface over $\mathbf{P}^{2}$ obtained by the projection from a triple or double point on the surface. This reduces the classification of the surfaces to the classification of branch loci satisfying certain conditions. Finally we derive some properties of the Hilbert schemes of some types of quintic surfaces.
\end{abstract}

1. Introduction. Algebraic surfaces over the complex number field $\mathbf{C}$ can be divided into four categories according to their Kodaira dimensions $(-\infty, 0,1,2)$. A surface is called of general type if the Kodaira dimension is 2. Much effort has been taken to give a classification of algebraic surfaces of general type. But there is no satisfactory result so far. Many authors studied the surfaces of general type with small invariants. In this paper we study the surfaces of general type in $\mathbf{P}^{3}$ with the smallest degree, i.e., quintic surfaces.

The easiest case is the quintic surfaces without essential singularities. Any such surface has the geometric invariants $p_{g}=4, q=0$ and $K^{2}=5$. All such surfaces form a Zariski open subset of $\mathbf{P}^{55}$. We will analyze the singular quintic surfaces and give a complete classification of the quintic surfaces of general type over $\mathbf{C}$.

The materials are organized as follows:

$\$ 2$ gives the background materials that we will use later on. Most results are well known and presented without proof.

In $\$ 3$ we give some formulae concerning double points. The key results are Theorems 3.3 and 3.5, which give a concrete description of the fundamental cycles of double points. Based on the formulae in this section we are able to calculate the geometric invariants of most singular quintic surfaces.

$\S 4$ gives all possible normal quintic surfaces of general type with one triple point as its only essential singularity.

In $\$ 5.1$ we prove that if a normal quintic surface has a double point with geometric genus greater than 1 then it is not a surface of general type. Then in $\S 5.2$

Received by the editors November 15, 1984.

1980 Mathematics Subject Classification. Primary 14J20. 
we give all possible quintic surfaces of general type with an elliptic double point as its only essential singularity.

In $\S 6$ we give all the remaining normal quintic surfaces of general type.

In $\S 7$ we show that quintic surfaces of general type must be normal. Note that a normal quintic surface with a quadruple point is a rational surface and that a quintic surface with a 5-tuple point is a cone, which is birational to a ruled surface. Therefore $\$ \S 4-6$ virtually give all quintic surfaces of general type with some essential singularities. As a by-product we prove that all quintic surfaces of general type are regular surfaces.

In $\S \S 8-10$ we give some description of the families of quintic surfaces. Unfortunately we are only able to handle some easy cases. For the surfaces with some bad double points the families are still unclear to me.

This paper comprises part of our thesis, written under the direction of M. Artin, to whom we here express our appreciation.

We thank the referee for his or her comments and suggestions.

2. Preliminaries. Throughout this paper the base field is always assumed to be the complex number field $\mathbf{C}$.

Let $S$ be a nonsingular compact surface. We denote the canonical divisor of $S$ by $K_{S}$ or simply $K$. For $n \geqslant 1$, let

$$
P_{n}=P_{n}(S)=\operatorname{dim} H^{0}\left(S, \mathcal{O}_{S}\left(n K_{S}\right)\right)
$$

be the $n$th plurigenus of $S$; if $n=1$, we write $p_{g}(S)$ instead of $P_{1}(S)$. The complete linear system $\left|n K_{S}\right|$ defines a rational map $\phi_{|n K|}: S \rightarrow \mathbf{P}^{P_{n}-1} . S$ is called a surface of general type if $\phi_{|n K|}$ is a birational morphism onto its image for some $n \geqslant 1$. The following lemma is simple but very useful.

LEMMA 2.1. If $S$ is a minimal surface of general type, then $A K \geqslant 0$ for any effective divisor $A$.

Proof. We may assume that $A$ is irreducible. Since $n K$ is linearly equivalent to some effective divisor for some $n, A K<0$ would imply that $A(n K)<0$ and hence $A^{2}<0$. By the Adjunction Formula we have $2 p_{a}(A)-2=A^{2}+A K \leqslant-2$. Since the arithmetic genus $p_{a}(A)$ is nonnegative, we have $p_{a}(A)=0$ and $A^{2}=A K=-1$. Hence $A$ is an exceptional curve. That contradicts the assumption that $S$ is minimal.

There is a criterion for surfaces of general type.

THEOREM 2.2 [11]. A minimal surface $S$ is of general type if and only if $K_{S}^{2} \geqslant 1$, $P_{2} \geqslant 2$.

There is also a formula for computing plurigenus.

THEOREM 2.3 [11]. If $S$ is a minimal surface of general type and $n \geqslant 2$, then the $n$th plurigenus $P_{n}$ of $S$ is given by

$$
P_{n}=\frac{1}{2} n(n-1) K^{2}+\chi(S) .
$$

Moreover, $\chi(S) \geqslant 1$. 
Isolated singularities. Let $p$ be a normal singularity on a surface $V$ which is not necessarily compact. We assume that $p$ is the only singularity on $V$. Let $\pi: M \rightarrow V$ be the minimal resolution of $V$. The set $A=\pi^{-1}(p)$ is called the exceptional set. Let $A=\bigcup A_{i}, 1 \leqslant i \leqslant n$, be the decomposition of $A$ into irreducible components. If we require that $A$ has the normal crossings, then $\pi$ is called the minimal good resolution.

THEOREM 2.4 [13]. The intersection matrix $\left(A_{i} A_{j}\right)$ is negative definite.

A cycle (or divisorial cycle) $D$ on $A$ is an integral combination of the $A_{i}$ 's. There is a natural partial ordering, denoted by $<$, between cycles. We only consider cycles $D \geqslant 0$. If $D=\sum d_{i} A_{i}, \sum d_{i}$ is called the degree of $D$.

The Riemann-Roch Theorem implies the useful formula

$$
\chi(B+C)=\chi(B)+\chi(C)-B C
$$

for any two cycles $B$ and $C$. If $B=\sum b_{i} A_{i}, C=\sum c_{i} A_{i}$, we will denote the cycle $\sum \min \left(b_{i}, c_{i}\right) A_{i}$ by $B \wedge C$. There is a weighted dual graph associated with each cycle (cf. $[12,18])$.

For any subvariety $B$ of $A$, there is a unique cycle $Z_{B}$ satisfying

(i) $\operatorname{Supp} Z=B$;

(ii) $A_{i} Z_{B} \leqslant 0$ for all $A_{i} \leqslant B$;

(iii) $Z_{B}$ is minimal with respect to these two properties

(cf. [2]). Such a cycle is called the fundamental cycle in the sense of M. Artin. We denote $Z_{A}$ by $Z$. The cycle $Z$ may be obtained via a computational sequence:

$$
Z_{0}=0, Z_{1}=A_{i_{1}}, Z_{2}=Z_{1}+A_{i_{2}}, \ldots, Z_{j}=Z_{j-1}+A_{i_{j}}, \ldots, Z_{l}=Z_{l-1}+A_{i_{l}}=Z
$$

where $A_{i_{1}}$ is arbitrary and $A_{i_{j}} Z_{j-1}>0$ for $1<j \leqslant l$.

The number $h=\operatorname{dim} H^{0}\left(V, R^{1} \pi_{*}\left(\mathcal{O}_{M}\right)\right)$ is called the geometric genus of $p$. Since $H^{1}(X, \mathscr{F})=0$ for any affine scheme $X$ and coherent sheaf $\mathscr{F}$, the sheaf $R^{1} \pi_{*}\left(\mathcal{O}_{M}\right)$ is concentrated at the point $p$. If $h=0, p$ is called a rational singularity.

Applying the Five-Term Sequence Theorem [15, p. 304] to the direct image functor $\pi_{*}$ and global section functor $\Gamma$, we have an exact sequence

$$
\begin{aligned}
0 & \rightarrow H^{1}\left(V, \mathcal{O}_{V}\right) \rightarrow H^{1}\left(M, \mathcal{O}_{M}\right) \rightarrow H^{0}\left(V, R^{1} \pi_{*} \mathcal{O}_{M}\right) \\
& \rightarrow H^{2}\left(V, \mathcal{O}_{V}\right) \rightarrow H^{2}\left(M, \mathcal{O}_{M}\right) \rightarrow 0 .
\end{aligned}
$$

In particular, $\operatorname{dim} H^{1}\left(M, \mathcal{O}_{M}\right)=\operatorname{dim} H^{0}\left(V, R^{1} \pi_{*} \mathcal{O}_{M}\right)$ if $V$ is affine.

From (2.3) we have

$$
\chi(V)=\chi(M)+h .
$$

If a surface $V$ has finite number of isolated singularities, then

$$
\chi(V)=\chi(M)+\sum_{p \text { sing }} h(p)
$$

where $M$ is the minimal resolution of $V$.

A singularity $p$ is called Gorenstein if the dualising sheaf $\omega$ is locally free at $p$. It was shown in [15] that a normal singularity on a surface is Gorenstein if and only if $K$ is linearly equivalent to some $H-K^{\prime}$, where $H, K^{\prime}$ are effective divisors on $M$ such that $H$ does not meet $A$ and $\operatorname{Supp}\left(K^{\prime}\right) \subseteq A$. The effective divisor $K^{\prime}$ is called the canonical cycle. If a neighborhood of $p$ can be embedded in $\mathbf{C}^{3}, p$ is Gorenstein. 
Definition 2.5. A cycle $D>0$ is rational if $\chi(D)=1$, elliptic if $\chi(D)=0$, minimally elliptic if $\chi(D)=0$ and $\chi(C)>0$ for all cycles $C$ such that $0<C<D$. Let $Z$ be the fundamental cycle of an isolated surface singularity $p ; p$ is called a rational (weakly elliptic, minimally elliptic) point if $Z$ is rational (elliptic, minimally elliptic).

Among all surface singularities the simplest ones are rational double points. They are classified into the following five types (cf. $[2,3])$ :

$$
\begin{array}{ll} 
& \text { representative equation } \\
A_{n} & x^{2}+y^{2}+z^{n+1}=0 \quad(n \geqslant 1) \\
D_{n} & x^{2}+y\left(y^{n-2}+z^{2}\right)=0 \quad(n \geqslant 4) \\
E_{6} & x^{2}+y^{3}+z^{4}=0 \\
E_{7} & x^{2}+y^{3}+z^{3} y=0 \\
E_{8} & x^{2}+y^{3}+z^{5}=0
\end{array}
$$

dual graph

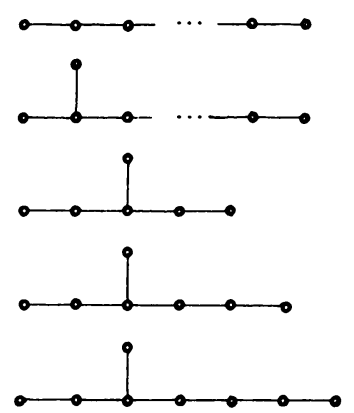

A singularity is called essential if it is not a rational double point. A surface is called essentially nonsingular if all singularities are rational double points.

The minimally elliptic singularities have already been classified by Laufer $[12,15$, 19]. There is a complete list of all possible weighted dual graphs for them [18]. We will use the following

THEOREM 2.6 [12]. Let $\pi: M \rightarrow V$ be the minimal resolution of a surface singularity p. Then $p$ is minimally elliptic if and only if $A_{i} Z=-A_{i} K_{M}$ for all irreducible components $A_{i}$ in $A$.

In particular, if $p$ is a minimally elliptic point, then the fundamental cycle $Z$ is the canonical cycle. We also know that $\operatorname{dim} H^{0}\left(V, R^{1} \pi_{*} \mathcal{O}_{M}\right)=1$.

Now suppose that $p$ is a weakly elliptic singularity. There is a unique minimally elliptic cycle $E$ in the sense that $E \leqslant D$ for all elliptic cycles $D$ [12, Proposition 3.2, p. 1261].

For Gorenstein weakly elliptic points, Yau [19] gave a method to compute the canonical cycle by using a so-called elliptic sequence. Here we give a proof by considering minimal resolution instead of minimal good resolution.

The following definition and theorem are due to Yau (cf. [19]).

Definition 2.7. Let $A$ be the exceptional set of the minimal resolution $\pi: M \rightarrow V$ where $V$ is a normal surface with $p$ as its only weakly elliptic singularity. If $E Z<0$, we say that the elliptic sequence is $\{Z\}$. Suppose $E Z=0$. Let $B_{1}$ be the maximal connected subvariety of $A$ such that $B_{1} \supseteq \operatorname{Supp} E$ and $A_{i} Z=0$ for all $A_{i} \subseteq B_{1}$. Since $Z^{2}<0, B_{1}$ is properly contained in $A$. Suppose $Z_{B_{1}} E=0$. Let $B_{2}$ be the maximal connected subvariety of $B_{1}$ such that $B_{2} \supseteq \operatorname{Supp} E$ and $A_{i} Z_{B_{1}}=0$ for all $A_{i} \subseteq B_{2}$. For the same reason as above, $B_{2}$ is properly contained in $B_{1}$. Continuing 
this process, we finally obtain $B_{m}$ with $Z_{B_{m}} E<0$. We call $\left\{Z_{B_{0}}=Z, Z_{B_{1}}, \ldots, Z_{B_{m}}\right\}$ the elliptic sequence of $p$.

THEOREM 2.8. Suppose the weakly elliptic point $p$ is Gorenstein and not minimally elliptic. Then

(i) $E Z_{B_{i}}=0$ for all $i<m$;

(ii) $Z_{B_{m}}=E$;

(iii) the canonical cycle $K^{\prime}$ is given by

$$
K^{\prime}=\sum_{i=0}^{m} Z_{B_{i}} .
$$

Proof. Write $K_{M} \sim H-K^{\prime}$ where $H$ does not meet $A$. We have $-A_{i} K^{\prime}=A_{i} K_{M}$ $\geqslant 0$ since $M$ is minimal. Hence $K^{\prime} \geqslant Z$ by the definition of fundamental cycle. So $K^{\prime}=Z+D$, with $D \geqslant 0$. Since $p$ is not minimally elliptic, $D>0$. Decompose $D=D_{1}+\cdots+D_{t}$ in such a way that $\operatorname{Supp} D_{i}$ is a connected component of Supp $D$. Then $K Z=-Z^{2}-Z D$ and $\chi(Z)=0$ imply that $Z D=0$, whence $Z D_{i}=0$ for each $i$. On the other hand, $D_{i} K=-D_{i} Z-D_{i}^{2}=-D_{i}^{2}$ implies that $\chi\left(D_{i}\right)=0$ for each $i$. Thus $t=1$ and $E \leqslant D$.

Let $B_{1}=\operatorname{Supp} D$. If $A_{i}$ is an irreducible component of $A$ such that $A_{i} \nless D$ and $A_{i} Z=0$, then $-A_{i} D=A_{i} K \geqslant 0$, which implies that $A_{i} D=0$. Hence $B_{1}$ is the maximal connected subvariety of $A$ such that $B_{1} \supseteq \operatorname{Supp} E$ and $A_{i} Z=0$ for all irreducible components $A_{i}$ of $B_{1}$.

For any irreducible component $A_{i}$ of $B_{1}, A_{i} D=-A_{i} K \leqslant 0$. So $D \geqslant Z_{B_{1}}$. Write $K^{\prime}=Z+Z_{B_{1}}+F$. If $Z_{B_{1}}=E$ and $F=0$, we are done. Suppose that $Z_{B_{1}} \neq E$ and $F=0$. Since $-2 \chi\left(Z_{B_{1}}\right)=Z_{B_{1}}^{2}-Z_{B_{1}} K^{\prime}=0, E<Z_{B_{1}}$. Write $Z_{B_{1}}=E+D$. Then $\chi(D)=1$. We have $E D=\chi(E)+\chi(D)-\chi\left(Z_{B_{1}}\right)=1$ by (2.2). On the other hand, $0=E^{2}+E K=E^{2}-E Z_{B_{1}}=-E D$ leads to a contradiction. Hence it remains to consider the case $Z_{B_{1}} \neq E$ and $F \neq 0$. The same argument as above shows that $B_{2}=\operatorname{Supp} F$ is the maximal connected subvariety of $A$ such that $B_{2}$ contains Supp $E$ and $A_{i} Z_{B_{1}}=0$ for all irreducible components $A_{i}$ of $B_{2}$.

We can continue this process to get a finite sequence $Z=B_{0}>B_{1}>\cdots>B_{m}$ and (2.6) is valid with $Z_{B_{m}}=E$.

A surface is called a Gorenstein surface if all its singularities are Gorenstein.

THEOREM 2.9 [6, p. 311]. Let $X$ be a complete algebraic Gorenstein surface with $p_{g}=0$, and let $\pi: \tilde{X} \rightarrow X$ be a resolution of singularities. Suppose that $\tilde{X}$ is not ruled. Then either

(a) $X$ is essentially nonsingular, or

(b) $X$ has exactly one minimally elliptic singularity of type E1, and every other singular point is a rational double point.

Case (b) does not occur if $X$ is of general type.

In Theorem 2.9, a minimally elliptic singularity of type E1 means that the exceptional divisor of that singularity is nonsingular.

Double covers. Let $f: X \rightarrow Y$ be a surjective holomorphic map of degree 2 between nonsingular surfaces $X$ and $Y$. Let $R$ be the ramification divisor of $f$. If $f$ is 
locally defined by $f\left(z_{1}, z_{2}\right)=\left(w_{1}, w_{2}\right)$ where $z_{1}, z_{2}$ and $w_{1}, w_{2}$ are local coordinates of $X$ and $Y$ respectively, then $R$ is the divisor of zeros of the Jacobian determinant $\partial\left(w_{1}, w_{2}\right) / \partial\left(z_{1}, z_{2}\right)$. The direct image $B=f_{*} R$ is called the branch locus of $f$. Since $X$ is nonsingular, $B$ has no multiple components.

Conversely, let $B$ be an effective divisor on $Y$ without multiple components. Let $\left\{U_{i}\right\}$ be a finite open covering of $Y$ such that $B$ is defined by a local equation $b_{i}=0$ on each $U_{i}$. We assume that there is a line bundle $F$ with such transition functions $\left\{f_{i j}\right\}$ that $b_{i}=f_{i j}^{2} b_{j}$ on $U_{i} \cap U_{j}$. The line bundle $F$ gives rise to a divisor on $Y$, which we still denote by $F$. Then the condition $b_{i}=f_{i j}^{2} b_{j}$ means that $B \sim 2 F$. Let $w_{i}$ denote the fibre coordinates on $F$ over $U_{i}$. Then the equations $w_{i}^{2}-b_{i}=0$ define a subvariety $X^{\prime} . X^{\prime}$ is well defined since $w_{i}^{2}-b_{i}=f_{i j}^{2}\left(w_{j}^{2}-b_{j}\right)$. Moreover, since the branch locus $B$ has no multiple components, $X^{\prime}$ is a normal surface. $X^{\prime}$ is called the double cover of $Y$ with branch locus $B$ after Horikawa [9]. Denote the double covering map by $f^{\prime}: X^{\prime} \rightarrow Y$.

If $B$ is nonsingular then $X^{\prime}$ is nonsingular. Assume that $B$ has singular points. Let $q_{1}: Y_{1} \rightarrow Y$ be a blowing-up with center at a singular point $s_{1}$ of $B$ with multiplicity $m_{1}$. Set $B_{1}=q_{1}^{*} B-2\left[m_{1} / 2\right] E_{1}$ and $F_{1}=q_{1}^{*} F-\left[m_{1} / 2\right] E_{1}$, where $E_{1}=q_{1}^{-1}\left(s_{1}\right)$. The line bundle $F_{1}$ and the divisor $B_{1}$ satisfy the conditions stated in the last paragraph. Thus we can construct the double cover $f_{1}: X_{1}^{\prime} \rightarrow Y_{1}$ with branch locus $B_{1}$.

LEMMA 2.10. There is a birational holomorphic map $\sigma_{1}: X_{1}^{\prime} \rightarrow X^{\prime}$ such that the diagram

$\begin{array}{rll}X_{1}^{\prime} & \stackrel{\sigma_{1}}{\rightarrow} & X^{\prime} \\ f_{1} \downarrow & & \downarrow f^{\prime} \\ Y_{1} & \rightarrow & Y\end{array}$

commutes.

In fact, $X_{1}^{\prime} \stackrel{\sigma_{1}}{\rightarrow} X^{\prime}$ is the blowing-up of $X^{\prime}$ with center at $f^{\prime-1}\left(s_{1}\right)$ followed by normalization.

Proof. Let $\phi: Y \rightarrow Y_{1}$ be the inverse birational transformation of $q_{1}$. Since $\phi$ is an isomorphism from $Y \backslash\left\{S_{1}\right\}$ onto $Y_{1} \backslash E_{1}$ which carries the branch locus isomorphically to branch locus, there is a birational transformation $\psi: X^{\prime} \rightarrow X_{1}^{\prime}$ such that the diagram

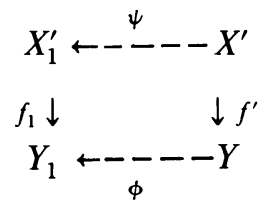

commutes. Let $\sigma_{1}$ be the inverse of $\psi$. The birational transformation $\psi$ restricted to $X^{\prime}-f^{\prime-1}\left(s_{1}\right)$ is an isomorphism. This implies that $\sigma_{1}$ has no fundamental points [17, V. 5.2]. It is clear that (2.7) commutes. Let $h: W \rightarrow X^{\prime}$ be the blowing-up of $X^{\prime}$ with center at $t_{1}=f^{\prime-1}\left(s_{1}\right)$ followed by normalization. By the universal property of blowing-up and normalization, there is a unique morphism $u: X_{1}^{\prime} \rightarrow W$ such that 
$h \circ u=\sigma_{1}$. It remains to show that $u$ is an isomorphism. By the universal property of the blowing-up there is a morphism $v: W \rightarrow Y_{1}$ such that $q_{1} \circ v=f^{\prime} \circ h$. Let $u^{\prime}$ be the birational transformation $\psi \circ h$. Then $f_{1} \circ u^{\prime}=v$ as rational maps. Since both $v$ and $f_{1}$ are finite, $u^{\prime}$ has no fundamental points. It is clear that both $u \circ u^{\prime}$ and $u^{\prime} \circ u$ are identity morphisms. Hence $u$ is an isomorphism.

If the branch locus $B_{1}$ has singular points, we repeat the above procedure, simply replacing $Y$ by $Y_{1}$. After a finite number of steps as shown in the diagram

\begin{tabular}{|c|c|c|c|c|c|c|c|c|c|c|}
\hline$X^{*}=X_{n}$ & $\rightarrow$ & $X_{n-1}$ & $\rightarrow$ & $\cdots$ & $\rightarrow$ & $X_{2}$ & $\rightarrow$ & $X_{1}$ & $\rightarrow$ & $X^{\prime}$ \\
\hline$f_{n} \downarrow$ & & $f_{n-1} \downarrow$ & & & & $\downarrow$ & & $f_{1} \downarrow$ & & $f^{\prime} \downarrow$ \\
\hline$Y_{n}$ & $\rightarrow$ & $Y_{n-1}$ & $\rightarrow$ & $\cdots$ & $\rightarrow$ & $Y_{2}$ & $\rightarrow$ & $Y_{1}$ & $\rightarrow$ & $Y$ \\
\hline
\end{tabular}

we get a nonsingular model $X^{*}=X_{n}$ of $X^{\prime}$. We call $X^{*}$ the canonical resolution of $X^{\prime}$. The nonsingular surface $X^{*}$ is not necessarily minimal.

Proposition $2.11[1$, p. 50; 9, p. 50]. Assume that $Y$ is compact in the above canonical resolution. Then

$$
\begin{gathered}
\chi\left(X^{*}\right)=\frac{1}{2} F\left(K_{Y}+F\right)+2 \chi(Y)-\frac{1}{2} \sum\left[m_{i} / 2\right]\left(\left[m_{i} / 2\right]-1\right), \\
K_{X^{*}}^{2}=2\left(K_{Y}+F\right)^{2}-2 \sum\left(\left[m_{i} / 2\right]-1\right)^{2}
\end{gathered}
$$

where each $m_{i}(i=1, \ldots, n)$ denotes the multiplicity of $B_{i-1}$ at the center of the blowing-up $q_{i}$ which appears in the process of the canonical resolution, and $F$ is half of the branch locus of $f^{\prime}$.

Double points. According to [10], a double point $p$ is analytically isomorphic to a double point given by an equation $x^{2}=f(y, z),(0,0)$ being a singular point of the power series $f(y, z)$. The double point is an isolated singularity if and only if $(0,0)$ is an isolated singularity of $f(y, z)=0$.

Let $m$ be the order of $f(y, z)$. By the Weierstrass Preparation Theorem we may write

$$
f(y, z)=u(y, z)\left(y^{m}+c_{1}(z) y^{m-1}+\cdots+c_{m-1}(z) y+c_{m}(z)\right)
$$

where $u(y, z)$ is a unit in the power series ring $\mathbf{C}[[y, z]]$ and $c_{1}(z), \ldots, c_{m}(z) \in \mathbf{C}[[z]]$. It can be shown (by using the theorem in [21], for instance) that the double point $p$ is analytically equivalent to that given by an equation

$$
x^{2}=y^{m}+d_{1}(z) y^{m-1}+\cdots+d_{m-1}(z) y+d_{m}(z)
$$

where each $d_{i}$ is a polynomial truncated from $c_{i}(z)$. Hence we may assume that $f(y, z)$ is a polynomial.

THEOREM 2.12. The geometric genus $h$ of a double point $x^{2}=f(y, z)$ is given by the formula

$$
h=\sum_{i} \frac{1}{2}\left[\frac{m_{i}}{2}\right]\left(\left[\frac{m_{i}}{2}\right]-1\right)
$$

where $m_{i}$ 's are the multiplicities which occur during the resolution of $(0,0)$ as defined in Proposition 2.11. 
Proof. Let $m$ be the degree of $f(y, z)$ and let $y, z, w$ be the homogeneous coordinates in $\mathbf{P}^{2}$. The zero locus $B^{\prime}$ of the homogeneous polynomial $w^{2[(m+1) / 2]} f(y / w, z / w)$ has no multiple components. Since $B^{\prime}$ has even degree, we can construct a double cover $S$ over $\mathbf{P}^{2}$ with branch locus $B^{\prime}$. Note that above the point $(0,0,1)$ is the double point $p$.

After making a canonical resolution of all singular points except $p$ we get a smocth surface $Y$ and an effective divisor $B$ such that $B \sim 2 F$ for some divisor $F$ and $B$ has only one singular point. Let $X$ be the double cover of $Y$ with branch locus $B . X$ has only one singular point, which is isomorphic to $p$. Let $X^{*}$ be the canonical resolution. Then (2.4) shows that

$$
\chi(X)=\chi\left(X^{*}\right)+h .
$$

Let $F^{*}$ be the completion of the line bundle $F$, by abuse of notation. Identify $Y$ with the zero section of $F^{*}$. There are on $F^{*}$ three exact sequences:

$$
\begin{aligned}
& 0 \rightarrow \mathcal{O}_{F^{*}}(-X) \rightarrow \mathcal{O}_{F^{*}} \rightarrow \mathcal{O}_{X} \rightarrow 0, \\
& 0 \rightarrow \mathcal{O}_{F^{*}}(-X) \rightarrow \mathcal{O}_{F^{*}}(-Y) \rightarrow \mathcal{O}_{Y}(-F) \rightarrow 0, \\
& 0 \rightarrow \mathcal{O}_{F^{*}}(-Y) \rightarrow \mathcal{O}_{F^{*}} \rightarrow \mathcal{O}_{Y} \rightarrow 0 .
\end{aligned}
$$

To see the second exact sequence, we can tensor product the last sequence with $\mathcal{O}_{F^{*}}(-Y)$ and use the facts that in $F^{*} X \sim 2 Y$ and $\left.Y\right|_{Y} \sim F$. So we have

$$
\begin{aligned}
\chi\left(X, \mathcal{O}_{X}\right) & =\chi\left(F^{*}, \mathcal{O}_{F^{*}}\right)-\chi\left(F^{*}, \mathcal{O}_{F^{*}}(-X)\right) \\
& =\chi\left(F^{*}, \mathcal{O}_{F^{*}}\right)-\chi\left(F^{*}, \mathcal{O}_{F^{*}}(-Y)\right)+\chi\left(Y, \mathcal{O}_{Y}(-F)\right) \\
& =\chi\left(Y, \mathcal{O}_{Y}\right)+\chi\left(Y, \mathcal{O}_{Y}(-F)\right) .
\end{aligned}
$$

Hence $\chi\left(X, \mathcal{O}_{X}\right)=2 \chi\left(Y, \mathcal{O}_{Y}\right)+\frac{1}{2} F\left(F+K_{Y}\right)$ by the Riemann-Roch Theorem.

Hence (2.11) and Proposition 2.11 yield

$$
\chi\left(X^{*}\right)=\chi(X)-\frac{1}{2} \sum\left[m_{i} / 2\right]\left(\left[m_{i} / 2\right]-1\right) .
$$

Therefore $h=\sum \frac{1}{2}\left[m_{i} / 2\right]\left(\left[m_{i} / 2\right]-1\right)$ by virtue of $(2.10)$.

Corollary. A double point $(0,0,0)$ defined by the equation $x^{2}=f(y, z)$ is a rational double point if and only if $(0,0)$ is not an m-tuple point of the locus $f(y, z)=0$ with $m>3$ and there is no infinitely near triple point of $f(y, z)=0$ above $(0,0)$.

3. Study of the fundamental cycle of a double point. Since the fundamental cycle was discovered by M. Artin [2] in 1966, it has been playing an important role in the study of surface singularities. In this section we study some basic properties of the fundamental cycle of an isolated double point.

First we prove some lemmas.

LemMA 3.1. Suppose $Z$ is a cycle with support on the exceptional set of an isolated surface singularity with the following two properties:

(i) $A_{i} Z \leqslant 0$ for all irreducible components $A_{i}$ of $Z$;

(ii) $Z^{2}=-1$.

Then $Z$ is the fundamental cycle of the singularity. 
Proof. If not, then $Z=Z^{\prime}+Z^{\prime \prime}$, where $Z^{\prime}$ is the fundamental cycle and $Z^{\prime \prime}>0$ by the first property of $Z$. The second property of $Z$ implies that

$$
-1=Z^{2}=\left(Z^{\prime}+Z^{\prime \prime}\right)^{2}=Z^{\prime 2}+Z^{\prime \prime 2}+2 Z^{\prime} Z^{\prime \prime} .
$$

But $Z^{\prime 2}<0, Z^{\prime \prime 2}<0$ by Theorem 2.4 , and $Z^{\prime} Z^{\prime \prime} \leqslant 0$ as $Z^{\prime}$ is the fundamental cycle. This leads to a contradiction.

LeMma 3.2. Let $X \stackrel{\pi}{\rightarrow} S$ be the resolution of an isolated singularity $p$ on a surface $S$. Let $\sigma: \tilde{X} \rightarrow X$ be the blowing-up of $X$ with center at some $q \in \pi^{-1}(p)$. Then the fundamental cycle $Z^{\prime}$ of $p$ on $\tilde{X}$ is given by $\sigma^{*}(Z)$, where $Z$ is the fundamental cycle of $p$ on $X$.

Proof. First of all it is obvious that $\operatorname{Do}^{*}(Z) \leqslant 0$ for any irreducible component $D$ of $\sigma^{*}(Z)$. So $Z^{\prime} \leqslant \sigma^{*}(Z)$. Write $Z^{\prime}=m E+\sum m_{i} \tilde{A}_{i}$, where $\tilde{A}_{i}$ is the proper transform of an irreducible component $A_{i}$ of $Z$ and $E$ is the exceptional curve of $\sigma$. Since $0 \geqslant E Z^{\prime}=-m+\sum m_{i}\left(E \tilde{A_{i}}\right)$, we have $\sigma^{*} \sigma_{*}\left(Z^{\prime}\right)=(m-k) E+\sum m_{i} \tilde{A}_{i}$ with $k \geqslant 0$. Thus $A_{i}\left(\sigma_{*} Z^{\prime}\right)=\tilde{A_{i}} Z^{\prime}-k \tilde{A_{i}} E \leqslant 0$ for all $A_{i}$. Hence $\sigma_{*}\left(Z^{\prime}\right)=Z$. Therefore $Z^{\prime}=\sigma^{*}(Z)$.

Let $p$ be a double point represented by the equation $x^{2}=g(y, z)$, where $g(y, z)$ is a formal power series in $y$ and $z$. Let $f: X \rightarrow \mathbf{C}^{2}$ be the double cover of $\mathbf{C}^{2}$ with branch locus $g(y, z)=0$. Then $f^{-1}(0,0)$ is isomorphic to $p$. Suppose that the canonical resolution of $p$ is given by the following diagram.

$$
\begin{array}{ccccccccccc}
X^{*}=X_{n} & \rightarrow & X_{n-1} & \rightarrow & \cdots & \rightarrow & X_{2} & \rightarrow & X_{1} & \rightarrow & X \\
\downarrow f_{n} & & \downarrow f_{n-1} & & & & \downarrow f_{2} & & \downarrow f_{1} & & \downarrow f \\
Y_{n} & \rightarrow & Y_{n-1} & \underset{q_{n}}{\rightarrow} & \cdots & \rightarrow & Y_{2} & \rightarrow & Y_{1} & \rightarrow & Y
\end{array}
$$

The nonsingular surface $X^{*}$ is a resolution of $X$, though it is not necessarily a minimal resolution. Define $E_{1}=q_{1}^{-1}(0,0)$ and $\bar{Z}=a^{*}\left(E_{1}\right)$, where $a=q_{2} \cdot q_{3} \cdots$ $q_{n} \cdot f_{n}$. Then $A_{i} \bar{Z}=\left(a_{*} A_{i}\right) E_{1} \leqslant 0$ for any irreducible component $A_{i}$ of $\bar{Z}$. Thus $Z \leqslant \bar{Z}$, where $Z$ is the fundamental cycle of $p$ on $X^{*}$.

A natural question arises: Is $Z$ equal to $\bar{Z}$ ? The answer is "yes" if $\operatorname{Ord}(g)$ is even, where $\operatorname{Ord}(g)$ is defined to be the minimal degree of the terms in $g(y, z)$. If $\operatorname{Ord}(g)$ is odd, then interesting things will happen. We will go through the details in the sequel.

THEOREM 3.3. If $\operatorname{Ord}(g)$ is even, then $\bar{Z}$ is the fundamental cycle of the double point $p$.

Proof. Let $\bar{E}_{1}$ be the proper transform of $E_{1}$ in $Y_{n}$ and let $D=f_{n}^{-1}\left(\bar{E}_{1}\right)$. Since $\operatorname{Ord}(g)$ is even, each irreducible component of $D$ is a simple component of $\bar{Z}$. Suppose the fundamental cycle $Z$ of $p$ is not equal to $\bar{Z}$. Then $Z^{\prime}=\bar{Z}-Z$ is a nonzero effective divisor. Since the support of $Z$ is the same as that of $\bar{Z}$, $Z^{\prime} \wedge D=\varnothing$. Hence

$$
Z^{\prime} \bar{Z}=Z^{\prime}\left(a^{*}\left(E_{1}\right)\right)=a_{*}\left(Z^{\prime}\right) E_{1}=0
$$


On the other hand, $-2=\bar{Z}^{2}=\left(Z+Z^{\prime}\right)^{2}=Z^{2}+2 Z Z^{\prime}+Z^{\prime 2}$ and $Z^{2}<0, Z^{\prime 2}<$ $0, Z Z^{\prime} \leqslant 0$ imply that $Z Z^{\prime}=0$. Hence $Z^{\prime} \bar{Z}=Z^{\prime}\left(Z+Z^{\prime}\right)=Z^{\prime 2}<0$, which contradicts (3.1).

Let $A=\bigcup_{i=0}^{m} A_{i}$ be the exceptional set of $p$ on $X^{*}$. If $\operatorname{Ord}(g)$ is odd, then $f_{n}^{-1}\left(\bar{E}_{1}\right)$ is always an irreducible rational curve. We may arrange $A_{i}$ in such a way that $A_{0}=f_{n}^{-1}\left(\bar{E}_{1}\right)$.

Lemma 3.4. If $\operatorname{Ord}(g)$ is odd, then there exists at most one cycle $Z^{\prime}=\sum k_{i} A_{i}<\bar{Z}$ such that

(i) $k_{0}=1$,

(ii) $A_{0} Z^{\prime}=-1, A_{i} Z^{\prime}=0$ for all $i>0$ such that $k_{i}>0$.

Actually, if such a $Z^{\prime}$ exists, then the fundamental cycle $Z$ of $p$ is given by $Z=\bar{Z}-Z^{\prime}$.

Proof. Suppose that $Z^{\prime}$ exists. Since $A_{0} \bar{Z}$ is equal to -1 , we have $A_{0}\left(\bar{Z}-Z^{\prime}\right)=0$ and $A_{i}\left(\bar{Z}-Z^{\prime}\right)=-A_{i} Z^{\prime} \leqslant 0$ for all $i \neq 0$.

On the other hand,

$$
Z^{2}=\left(\bar{Z}-Z^{\prime}\right)^{2}=-2-2 \bar{Z} Z^{\prime}+Z^{\prime 2}=-2-2 \bar{Z} Z^{\prime}-1 .
$$

By Theorem 2.4, we have $Z^{2}<0$. Hence

$$
\bar{Z} Z^{\prime}=\bar{Z} A_{0}=-1,
$$

so $Z^{2}=-1$. Therefore $Z=\bar{Z}-Z^{\prime}$ is the fundamental cycle of $p$ on $X^{*}$ by Lemma 3.1. Since the fundamental cycle is unique, $Z^{\prime}$ is unique if it exists.

Definition. Let $X^{*}$ be the canonical resolution of a double point $p$ defined by $x^{2}=g(y, z)$. Let $e=\operatorname{Ord}(g)$. If $e$ is odd and a cycle $Z^{\prime}$ satisfying the conditions in Lemma 3.4 exists, then $Z^{\prime}$ is called the redundancy cycle of $p$ on $X^{*}$. A double point is said to be of type I if $e$ is even or $p$ has no redundancy cycle, of type II if $e$ is odd and $p$ has a redundancy cycle, of type $\mathrm{II}_{\mathrm{a}}$ if the redundancy cycle is irreducible, of type $\mathrm{II}_{\mathrm{b}}$ if the redundancy cycle is reducible.

THEOREM 3.5. Let $X^{*}$ be the canonical resolution of an isolated double point $p$ defined by $x^{2}=g(y, z)$. Let $e=\operatorname{Ord}(g)$. Then

(i) $p$ is of type $\mathrm{II}_{\mathrm{a}}$ if and only if $e$ is odd and there is an infinitely near e-tuple point of the zero locus of $g(y, z)$ over $(0,0)$. In this case the redundancy cycle $Z^{\prime}$ is $A_{0}$;

(ii) The fundamental cycle $Z$ of $p$ on $X^{*}$ is given by

$$
Z= \begin{cases}\bar{Z}, & \text { if } p \text { is of type } \mathrm{I}, \\ \bar{Z}-Z^{\prime}, & \text { if } p \text { is of type II. }\end{cases}
$$

Proof. If $e$ is odd and there is an infinitely near $e$-tuple point of the zero locus of $g$ over $(0,0)$, then $E_{1} G=e$, where $G$ is the proper transform of the zero locus of $g$. The intersection point of $E_{1}$ and $G$ is an $e$-tuple point of $G$. Thus the proper transform of $E_{1}$ in $Y_{2}$ is a connected component of the branch locus of $f_{2}$. Hence $\bar{E}_{1}$ is in the branch locus of $f_{n}$ with $\bar{E}_{1}^{2}=-2$. So $A_{0}^{2}=-1$. The cycle $A_{0}$ satisfies the conditions in Lemma 3.4. Hence $p$ is of type $\mathrm{II}_{\mathrm{a}}$. Conversely, if $Z^{\prime}$ is irreducible, then $A_{0}=Z^{\prime 2}=-1$ show that $\bar{E}_{1}$ is in the branch locus and $\bar{E}_{1}^{2}=-2$. That can 
only happen when $e$ is odd and there is an infinitely near $e$-tuple point of the zero locus of $g$ over $(0,0)$. This proves (i).

As to (ii), it suffices to show that $e$ odd and $Z \neq \bar{Z}$ imply that $p$ is of type II. Suppose $Z \neq \bar{Z}$. Then $Z<\bar{Z}$. Write $\bar{Z}=Z+Z^{\prime}$ and $\bar{Z}=\sum c_{i} A_{i}, Z^{\prime}=\sum k_{i} A_{i}$. Since $e$ is odd, $c_{0}$ equals 2. Since $Z^{\prime 2}=-1$, there must be some component $A_{r}$ of $Z^{\prime}$ such that $A_{r} Z^{\prime}<0$. Since $A_{r} \bar{Z}=A_{r} Z+A_{r} Z^{\prime}<0, A_{r}$ must be $A_{0}$. Moreover, $A_{0} Z^{\prime}=-1, A_{0} Z=0$. Since $Z^{2}=-1$ and $A_{i} Z \leqslant 0$ for all $i$, there exists only one $A_{j}$ such that $A_{j} Z=-1$ and $A_{i} Z=0$ for all $i \neq j$. Since $A_{0} Z=0$, we infer that $j \neq 0$. We also have

$$
A_{i} Z^{\prime}=-A_{i} Z= \begin{cases}1 & \text { if } i=j \\ 0 & \text { if } i \neq j\end{cases}
$$

for all $i>0$. On the other hand, $-2=\bar{Z}^{2}=\left(Z+Z^{\prime}\right)^{2}=Z^{2}+2 Z Z^{\prime}+Z^{\prime 2}$ and $Z^{2}<0, Z^{\prime 2}<0, Z Z^{\prime} \leqslant 0$ imply that $Z Z^{\prime}=0$. So $A_{i} Z=0$ for all $i$ such that $k_{i} \neq 0$. Hence $A_{i} Z^{\prime}=A_{i} \bar{Z}-A_{i} Z=0$ for all $i>0$ such that $k_{i} \neq 0$. Therefore $Z^{\prime}$ is a redundancy cycle.

REMARK. Let $\tilde{X} \rightarrow X$ be the minimal resolution of the surface $X$ with an isolated double point $p$. Then there is a unique birational morphism from $X^{*}$ to $\tilde{X}$. Lemma 3.2 shows that the fundamental cycle $Z$ on $X^{*}$ is the total transform of the fundamental cycle of $p$ on $\tilde{X}$. Therefore both Euler characteristic and self-intersection number of the fundamental cycle remain unchanged.

COROllary 3.6. Let $S \rightarrow X$ be any resolution of a surface $X$ with an isolated double point $p$. Then

$$
Z^{2}= \begin{cases}-2 & \text { if } p \text { is of type } \mathrm{I}, \\ -1 & \text { if } p \text { is of type } \mathrm{II},\end{cases}
$$

where $Z$ is the fundamental cycle of $p$ on $S$.

Proof. By the remark, we may assume that $S=X^{*}$. If $p$ is of type $\mathrm{I}$, then $Z^{2}=\bar{Z}^{2}=2 E_{1}^{2}=-2$. If $p$ is of type II, then $Z^{2}=-1$ as shown in the proof of Theorem 3.5.

EXAMPLE. Let $p$ be a double point defined by

$$
x^{2}=\left(y^{2}+z^{4}\right)\left(y^{3}+z^{4}\right) \text {. }
$$

The fundamental cycle $Z$ on $X^{*}$ is given by the weighted dual graph

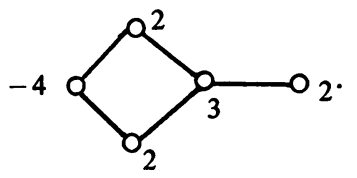

We can see that $Z=\bar{Z}$. Hence $p$ is a double point of type I. This example shows that the converse of Theorem 3.3 is not true.

THEOREM 3.7. Let $Z$ be the fundamental cycle of an isolated double point $p$ represented by $x^{2}=g(y, z)$. Let $e=\operatorname{Ord}(g)$. Then

$$
\chi(Z)= \begin{cases}2-\left[\frac{1}{2} e\right] & \text { if } p \text { is of type } \mathrm{I}, \\ \frac{1}{2}(3-e) & \text { if } p \text { is of type } \mathrm{II}_{\mathrm{a}},\end{cases}
$$




$$
\chi(Z) \geqslant 2-\left[\frac{1}{2} e\right] \text { if } p \text { is of type } \mathrm{II}_{\mathrm{b}} \text {. }
$$

Here $[\alpha]$ denotes the integer part of the real number $\alpha$.

Proof. By the Adjunction Formula,

$$
\begin{aligned}
\chi(\bar{Z}) & =-\frac{1}{2}\left(\bar{Z}^{2}+\bar{Z} K_{X^{*}}\right)=1-\frac{1}{2} E_{1}\left(2 K_{Y_{1}}+B_{1}\right) \\
& = \begin{cases}2-\frac{1}{2} e & \text { if } e \text { is even, } \\
2-\frac{1}{2}(e-1) & \text { if } e \text { is odd. }\end{cases}
\end{aligned}
$$

Here $B_{1}$ is the branch locus of $f_{1}$ and $K_{Y_{1}}$ is the canonical divisor of $Y_{1}$. Hence $\chi(Z)=\chi(\bar{Z})=2-\left[\frac{1}{2} e\right]$ if $p$ is of type $\mathrm{I}$.

If $p$ is of type $\mathrm{II}_{\mathrm{a}}$, then $\chi\left(Z^{\prime}\right)=1$ and $\left[\frac{1}{2} e\right]=\frac{1}{2}(e-1)$. Hence $\chi(Z)=\chi(\bar{Z})-$ $\chi\left(Z^{\prime}\right)+Z Z^{\prime}=2-\left[\frac{1}{2} e\right]-\chi\left(Z^{\prime}\right)=\frac{1}{2}(3-e)$.

If $p$ is of type $\mathrm{II}_{\mathrm{b}}$, then $A_{0}^{2}=-r<-1$. By Lemma 3.1, $Z^{\prime}$ is a fundamental cycle on its own support. Hence $\chi\left(Z^{\prime}\right) \leqslant 1$. Suppose $\chi\left(Z^{\prime}\right)=1$. Then either $Z^{\prime}$ would be the fundamental cycle of a rational double point or there would be a morphism from $X^{*}$ to a smooth surface so that $Z^{\prime}$ would contract to a point. Since $Z^{\prime 2}=-1, Z^{\prime}$ would not be the fundamental cycle of a rational double point. Thus $Z^{\prime}$ would contain an exceptional curve $C_{1}$ and a rational curve $C_{2}$ with $C_{2}^{2}=-2$ and $C_{1} C_{2}=1$. Then $f_{n}\left(C_{1}\right)$ would be a rational curve of self-intersection -2 in the branch locus and the map from $C_{2}$ onto $f_{n}\left(C_{2}\right)$ would be a double cover with two ramification points. That would mean that $f_{n}\left(C_{2}\right)$ would be an exceptional curve having two intersection points with the branch locus. But this is quite impossible for the construction of canonical resolution. Hence $\chi\left(Z^{\prime}\right)<1$. Therefore $\chi(Z)=\chi(\bar{Z})$ $-\chi\left(Z^{\prime}\right) \geqslant 2-[e / 2]$.

4. Normal quintic surfaces with a triple point. In this section we will prove the following theorem.

THEOREM 4.1. A normal quintic surface $S_{0}$ of general type which has a triple point $p$ as its only essential singularity must be one of the following three types:

(1) $p$ is a minimally elliptic point.

(2) The blowing-up of $S_{0}$ at $p$ has a minimally elliptic double point $q$ of type I and $S_{0}$ contains a line $L_{0}$ such that the proper transform of $L_{0}$ passes through $q$.

(3) The blowing-up of $S_{0}$ at $p$ has a minimally elliptic double point of type $\mathrm{II}_{\mathrm{a}}$.

These three types of surfaces are denoted by III, III-I' and III-II ${ }_{a}$.

The numerical invariants of the minimal models are:

$$
p_{g}=3, \quad q=0, \quad K^{2}=2 \quad \text { for III, }
$$

and

$$
p_{g}=2, \quad q=0, \quad K^{2}=1 \quad \text { for III-I' and III-II }{ }_{a} .
$$

Remark. The notation "'" indicates that the minimal resolution of $S_{0}$ is not a minimal surface.

4.1 Isolated hypersurface triple points. First of all, we study some general properties of a hypersurface triple point. 
Let $p=(0,0,0)$ be an isolated triple point on a hypersurface $S_{0} \subset \mathbf{A}^{3}$ defined by the equation

$$
f(x, y, z)=f_{3}(x, y, z)+f_{4}(x, y, z)+\cdots=0
$$

where $f_{i}(x, y, z)$ denotes the degree $i$ homogeneous part of $f(x, y, z)$ and $f_{3}(x, y, z)$ $\neq 0$. Let $\sigma: T \rightarrow \mathbf{A}^{3}$ be the blowing-up of $\mathbf{A}^{3}$ at $p$. Denote the proper transform of $S_{0}$ in $T$ by $S$. Let $E=\sigma^{-1}(p)$. Then $x, y, z$ can be regarded as the homogeneous coordinates of the plane $E$. Let $D_{i}$ be the plane curve defined by $f_{i}(x, y, z)=0$, where $i=3,4, \ldots$ Obviously $D_{3}=S \cap E$ can be one of the following curves:

(i) a nonsingular curve;

(ii) a rational curve with a node;

(iii) a rational curve with a cusp;

(iv) the union of a line and a conic with two distinct intersection points;

(v) the union of a line and a conic tangent to each other;

(vi) the union of three distinct nonconcurrent lines;

(vii) the union of three distinct concurrent lines;

(viii) the union of a double line and another line;

(ix) a triple line.

Proposition 4.2. The set of singular points of $S$ on $E$ is ( $\left.\operatorname{Sing} D_{3}\right) \cap D_{4}$, where Sing $D_{3}$ is the set of singular points of $D_{3}$.

Proof. The local equations of $S$ are

$$
\begin{aligned}
& f_{3}(1, y, z)+x f_{4}(1, y, z)+x^{2} f_{5}(1, y, z)+\cdots=0 \\
& f_{3}(x, 1, z)+y f_{4}(x, 1, z)+y^{2} f_{5}(x, 1, z)+\cdots=0 \\
& f_{3}(x, y, 1)+z f_{4}(x, y, 1)+z^{2} f_{5}(x, y, 1)+\cdots=0 .
\end{aligned}
$$

By the symmetry of these three equations, it suffices to discuss one of them, say (4.2). Note that $E$ is given by $x=0$. The Jacobi criterion gives the following conditions for a singular point of $S$ on $E$ :

$$
\begin{gathered}
f_{3}(1, y, z)=0, \quad \frac{\partial f_{3}}{\partial y}(1, y, z)=0, \\
\frac{\partial f_{3}}{\partial z}(1, y, z)=0, \quad f_{4}(1, y, z)=0,
\end{gathered}
$$

which is what we want to prove.

Proposition 4.3. If $S$ is nonsingular on $E$, then $p$ is a minimally elliptic singularity.

Proof. Without loss of generality, we may assume that $p$ is the only singularity of $S$. That means that $S$ is smooth. We have

$$
K_{T}+S \sim\left(\sigma^{*} K_{\mathbf{A}^{3}}+2 E\right)+\left(\sigma^{*} S_{0}-3 E\right) \sim \sigma^{*}\left(K_{\mathbf{A}^{3}}+S_{0}\right)-E .
$$

The canonical divisor $K$ of $S$ is linearly equivalent to the restriction of $K_{T}+S$ on $S$. Since $K_{\mathrm{A}^{3}}+S_{0}$ is linearly equivalent to a divisor away from $p$, the canonical cycle $K^{\prime}$ of $p$ in $S$ is the restriction of $E$ on $S$. By the Adjunction Formula, $\chi\left(K^{\prime}\right)=K^{\prime 2}$ $+K^{\prime}\left(-K^{\prime}\right)=0$. Hence $K^{\prime}$ is an elliptic cycle. Since the intersection of $E$ and $S$ is a plane cubic curve, $K^{\prime}$ must be a minimally elliptic cycle. Therefore $p$ is minimally elliptic. 
REMARK. Since rational double points are negligible, the proposition is still true even if $S$ has some rational double points on $E$.

Proposition 4.4. If $D_{3}$ is one of the curves (i), (ii), (iv) and (vi), then $p$ is a minimally elliptic point.

Proof. In cases (i), (ii), (iii) and (iv), $D_{3}$ has at most nodes as singularities. Let $\left(y_{1}, z_{1}\right)$ be a point satisfying $f_{3}\left(1, y_{1}, z_{1}\right)=0$. Let $y^{\prime}=y-y_{1}$ and $z^{\prime}=z-z_{1}$. Then $f_{i}(1, y, z)$ in $(4.2)$ can be written as $f_{i}^{\prime}\left(y^{\prime}, z^{\prime}\right)$ for $i=3,4,5, \ldots$. The order of $f_{3}^{\prime}\left(y^{\prime}, z^{\prime}\right)$ is either 1 or 2 . In the former case, $S$ is smooth at the point $x=0, y=y_{1}$, $z=z_{1}$. In the latter case the degree two part of $f_{3}^{\prime}\left(y^{\prime}, z^{\prime}\right)$ is the product of two different linear factors as $D_{3}$ has at most nodes. Hence the point $x=0, y=y_{1}$, $z=z_{1}$ is a rational double point in that case. The same discussion applies to the equations (4.3) and (4.4). Hence $p$ is a minimally elliptic point by Proposition 4.3 and the remark.

4.2 Proof of The Theorem. Now we return to the quintic surface $S_{0}$. Let $S_{0}$ be defined by $f(x, y, z)=0$, where $f(x, y, z)=f_{3}(x, y, z)+f_{4}(x, y, z)+f_{5}(x, y, z)$. We still use the notations in $\S 4.1$.

Proposition 4.5. If $S$ is not normal and if $p$ is the only essential singularity of $S_{0}$, then the minimal model of $S_{0}$ is a $K 3$ surface.

Proof. Proposition 4.2 implies that $S$ is not normal if and only if case (viii) or (ix) happens and $f_{4}(x, y, z)$ vanishes along the double or triple line of $D_{3}$. We may assume that $f_{3}(x, y, z)=y^{2} z$ or $y^{3}$ and $y \mid f_{4}(x, y, z)$. Write

$$
f_{4}(x, y, z)=y g_{3}(x, y, z) \text {. }
$$

Then (4.2) becomes

$$
y^{2} z+x y g_{3}(1, y, z)+x^{2} f_{5}(1, y, z)=0,
$$

or

$$
y^{3}+x y g_{3}(1, y, z)+x^{2} f_{5}(1, y, z)=0 \text {. }
$$

The line $L: y=0, x=0$ is a double line of $S$. Let $\sigma^{\prime}: T^{\prime} \rightarrow T$ be the blowing-up of the threefold $T$ along $L$ and let $S^{\prime}$ be the proper transform of $S$. Let $F$ be the exceptional divisor of $\sigma^{\prime}$. It is easy to check that $S^{\prime}$ has no essential singularities. We have

$$
K_{T^{\prime}}+S^{\prime} \sim \sigma^{\prime *}\left(K_{T}+S\right)-F \sim \sigma^{\prime *}\left(\sigma^{*}\left(K_{\mathbf{P}^{3}}+S_{0}\right)-E\right)-F .
$$

The divisor $K_{\mathbf{P}^{3}}+S_{0}$ is linearly equivalent to a hyperplane in $\mathbf{P}^{3}$, so the canonical system $\left|K_{S^{\prime}}\right|$ is cut out by the plane $H_{0}$ in $\mathbf{P}^{3}$ passing through $p$ such that the proper transform $H$ of $H_{0}$ in $T$ passes through $L$. Actually $H_{0}$ is given by $y=0$. Thus $H_{0} \cap S_{0}$ is the union of five concurrent lines $L_{1}, \ldots, L_{5}$. We discuss the following different cases:

(i) (General case) If $L_{1}, \ldots, L_{5}$ are distinct, then a direct calculation shows that $S_{0}$ is smooth on $H_{0}-\{p\}$. Let $L_{1}^{\prime}, \ldots, L_{5}^{\prime}$ be the proper transforms of $L_{1}, \ldots, L_{5}$ in $S^{\prime}$. Then $K_{S^{\prime}} \sim L_{1}^{\prime}+\cdots+L_{5}^{\prime}$ and $S^{\prime}$ is smooth along $L_{i}^{\prime}$ for $1 \leqslant i \leqslant 5$. Obviously $L_{1}^{\prime}, \ldots, L_{5}^{\prime}$ are pairwise disjoint. Hence $L_{1}^{\prime}, \ldots, L_{5}^{\prime}$ are exceptional curves of first 
kind by the Adjunction Formula. Therefore the minimal model of $S_{0}$ is a $K 3$ surface.

(ii) (Special case) Suppose $L_{1}, \ldots, L_{5}$ are not distinct, say $L_{1}=L_{2}=\cdots=L_{r}$ and $L_{i} \neq L_{1}$ for $i>r$. That means that there is a linear form $\lambda(x, z)$ such that $\lambda^{r}(x, z) \mid f_{5}(x, 0, z)$ and $\lambda^{r+1}(x, z)+f_{5}(x, 0, z)$. Under the circumstances, $S^{\prime}$ has a rational double point of type $A_{r-1}$ on $L_{1}^{\prime}$. Let $\tilde{\sigma}: \tilde{S} \rightarrow S^{\prime}$ be the minimal resolution of $S^{\prime}$. Then the effective canonical divisor of $\tilde{S}$ contains a chain

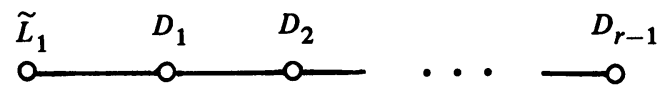

as one of its connected components, where $\tilde{L}_{1}$ is the proper transform of $L_{1}^{\prime}$ and $D_{1}, \ldots, D_{r-1}$ are rational curves with self-intersection -2 . Suppose that $K_{\tilde{S}} \sim r \tilde{L}_{1}+$ $m_{1} D_{1}+\cdots+m_{r-1} D_{r-1}+G$, where $G$ is disjoint from $\tilde{L}_{1}+\sum D_{i}$. Then the Ad junction Formula yields the following equalities:

$$
\begin{aligned}
(r+1) \tilde{L}_{1}^{2}+m_{1} & =-2, \\
-2 m_{1}+r+m_{2} & =0 \\
-2 m_{2}+m_{1}+m_{3} & =0 \\
& \vdots \\
-2 m_{r-1}+m_{r-2} & =0
\end{aligned}
$$

Adding all these together we get

$$
(r+1) \tilde{L}_{1}^{2}+r-m_{r-1}=-2 .
$$

The equality (4.8) implies $\tilde{L}_{1}^{2}<0$ while (4.9) implies $\tilde{L}_{1}^{2}>-2$. Hence $\tilde{L}_{1}^{2}=-1$. Thus $\left\{\tilde{L}_{1}, D_{1}, \ldots, D_{r-1}\right\}$ is a chain of exceptional divisors of first kind. The same arguments apply to other components of $\left|K_{\tilde{S}}\right|$. Therefore $\tilde{S}$ is a $K 3$ surface.

REMARK. Now that the minimal model of $S_{0}$ is a $K 3$ surface under the conditions of the proposition, then we would like to know the curves on it. Take a generic line $L_{0}$ in $\mathbf{P}^{3}$ passing through $p$. Let $H_{0}$ be a generic hyperplane passing through $L_{0}$. Then $H_{0} \cap S_{0}$ is a quintic curve with a triple point plus an infinitely near double point. Hence all hyperplanes passing through $L_{0}$ give rise to a pencil of genus 2 curves with two base points on the minimal model of $S_{0}$.

Proposition 4.6. If $S$ is not normal and if there is an essential singularity other than $p$, then the minimal model of $S_{0}$ is a ruled surface.

Proof. Following the same arguments as in the proof of Proposition 4.5, we see that $K_{\tilde{S}} \sim-D \neq 0$ where $\tilde{S}$ is a minimal model of $S_{0}$ and $D$ is an effective divisor with support on the union of the exceptional divisors of all essential singularities other than $p$. Hence $\tilde{S}$ is a ruled surface.

Proof of Theorem 4.1. Denote the minimal resolution of $S_{0}$ by $\tilde{S}$.

We first compute the numerical invariants for the surfaces of type III. If $p$ is a minimally elliptic point, then $\left|K_{\tilde{S}}\right|$ is cut out by all hyperplanes passing through $p$. Thus the only possible fixed components of $\left|K_{\tilde{S}}\right|$ are in the exceptional divisor $C$ of $p$. Hence $\tilde{S}$ is minimal. Therefore $p_{g}=3, K_{\tilde{S}}^{2}=5+C^{2}=5-3=2$. Since $\chi\left(\mathcal{O}_{\tilde{S}}\right)$ $=\chi\left(\mathcal{O}_{S_{0}}\right)-1=4$, we have $q=0$. 
Remember that there are nine cases for $D_{3}$. In cases (i), (ii), (iv) and (vi), $p$ is minimally elliptic by Proposition 4.4. Henceforth we discuss all the remaining cases.

(iii) We may assume that $f_{3}(1, y, z)=y^{2}+z^{3}$. Then (4.2) can be written as

$$
y^{2}+z^{3}+x f_{4}(1, y, z)+x^{2} f_{5}(1, y, z)=0 .
$$

Since $f_{4}(1, y, z)$ or $f_{5}(1, y, z)$ must contain at least one nonzero monomial among 1 , $y$ and $z, S$ has at most a rational double point on $D_{3}$. Hence $p$ is minimally elliptic by the remark to Proposition 4.4 .

(v) We may assume that $f_{3}(1, y, z)=y\left(y-z^{2}\right)$. Then (4.2) becomes

$$
y^{2}-y z^{2}+x f_{4}(1, y, z)+x^{2} f_{5}(1, y, z)=0 .
$$

If either $f_{4}(1, y, z)$ contains a nonzero monomial from $1, y, z, z^{2}$ or $f_{5}(1, y, z)$ contains a nonzero monomial from $1, z$, then $S$ has at most a rational double point on $D_{3}$. In that case $p$ is minimally elliptic. Otherwise $S$ has a minimally elliptic double point $q$ of type I. The line $L_{0}: y=0, z=0$ is on $S_{0}$ and the proper transform $L$ of $L_{0}$ passes through $q$. Let $S_{1}$ be the minimal resolution of $S$. Then $\left|K_{S_{1}}\right|$ is cut out by all hyperplanes in $\mathbf{P}^{3}$ passing through $L_{0}$. Hence $p_{g}(\tilde{S})=2$. Let $L_{1}$ be the proper transform of $L$ in $S_{1}$. Then $L_{1}$ is the only fixed component of $\left|K_{S_{1}}\right|$. Since $L$ meets the canonical cycle of $p$ at least twice, $L_{1} K_{S_{1}}<0$. Hence $L_{1}$ is an exceptional curve of the first kind. Let $\eta: S_{1} \rightarrow \tilde{S}$ be the contraction of $L_{1}$. Then $\tilde{S}$ is the minimal model of $S_{1}$. Since $K_{S_{1}}^{2}=5-3-2=0$, we have $K_{\tilde{S}}^{2}=1$. Since the geometric genus of $p$ is equal to 2 , we have $\chi\left(\mathcal{O}_{\tilde{S}}\right)=\chi\left(\mathcal{O}_{S_{0}}\right)-2=3$. Hence $q(\tilde{S})=0$.

(vii) We may assume that $f_{3}(x, y, z)=y z(y+z)$. Then (4.2) takes the form

$$
y z(y+z)+x f_{4}(1, y, z)+x^{2} f_{5}(1, y, z)=0 .
$$

If either $f_{4}(1, y, z)$ contains a nonzero monomial from $1, y, z$ or $f_{5}(1, y, z)$ has a nonzero constant term, then $S$ has at most a rational double point on $D_{3}$. In that case, $p$ is minimally elliptic.

Otherwise, (4.10) gives rise to a triple point $q$ on $S$. The degree 3 part in (4.10) is $y z(y+z)+x^{2}(\lambda y+\mu z)$, where $\lambda$ and $\mu$ cannot be zero at the same time. To finish the discussion of this case we prove the following proposition.

Proposition 4.7. If $D_{3}$ is the union of three concurrent lines intersecting at a triple point $q$ of $S$, then the minimal model of $S_{0}$ is an elliptic surface.

Proof. Proposition 4.4 implies that $q$ is a minimally elliptic triple point. Let $S_{1}$ be the minimal resolution of $S$. By the same arguments as above, $\left|K_{S_{1}}\right|$ is cut out by all the hyperplanes passing through a line $L_{0}$ in $\mathbf{P}^{3}$, and the proper transform $L_{1}$ of $L_{0}$ in $S_{1}$ is the only exceptional divisor of the first kind in $S_{1}$. Let $\eta: S_{1} \rightarrow \tilde{S}$ be the blowing-down of $L_{1}$. Then $\tilde{S}$ is a minimal model of $S_{0}$. Since $p_{g}(\tilde{S})=2, K_{\tilde{S}}^{2}=5-$ $3-3+1=0, \tilde{S}$ is an elliptic surface.

Continuation of the proof of Theorem 4.1.

(viii) We may assume that $f_{3}(x, y, z)=y^{2} z$. Then (4.2) becomes

$$
y^{2} z+x f_{4}(1, y, z)+x^{2} f_{5}(1, y, z)=0 .
$$


If $y \mid f_{4}(1, y, z)$, then $S$ is not normal and Proposition 4.5 implies that the minimal model of $S_{0}$ is a $K 3$ surface. Hence we may assume $y+f_{4}(1, y, z)$. By Proposition 4.2, all the singular points of $S$ on $D_{3}$ are located on the line $L: y=0, x=0$ on $S$, which are determined by the roots of $f_{4}(1,0, z)=0$. In other words, it suffices to investigate the point $q$ : $x=0, y=0, z=\zeta$, where $\zeta$ is a root of $f_{4}(1,0, z)=0$. Note that $\zeta$ might be $\infty$ ! But that does not matter, for we can use (4.4) instead of (4.2) in that case.

If $\zeta$ is a simple root of $f_{4}(1,0, z)=0$, then $q$ is a rational double point. If $\zeta$ is a double root of $f_{4}(1,0, z)=0$ and $\zeta \neq 0$, then $q$ is also a rational double point. That implies that $S$ has at most one essential singularity.

If $f_{5}(1,0,0) \neq 0$ or $y^{2}+f_{4}(1, y, 0)$, then $q$ is a rational double point. Hence an essential singularity is given by one of the following two sets of conditions:

(a) $\zeta=0, z^{2}\left|f_{4}(1,0, z), y^{2}\right| f_{4}(1, y, 0), f_{5}(1,0,0)=0$;

(b) $\zeta \neq 0,(z-\zeta)^{3}\left|f_{4}(1,0, z), y^{2}\right| f_{4}(1, y, \zeta),(z-\zeta)^{2}+f_{5}(1,0, \zeta)$.

In case (a) $f_{5}(1, y, z)$ must contain a nonzero monomial from $y$ and $z$ and $f_{4}(1,0, z) \neq 0$. Hence $S$ has a minimally elliptic triple point. Therefore the minimal model of $S_{0}$ is an elliptic surface. Actually the hyperplanes in $\mathbf{P}^{3}$ passing through the line $L_{0}: y=0, z=0$ cut out a pencil of elliptic curves.

In case (b) $S$ has a minimally elliptic double point of type $I$. Let $\tilde{L}$ be the proper transform of the line $L_{0}: y=0, z=0$ in the minimal resolution $\tilde{S}$ of $S_{0}$. Then $\tilde{L}$ is an exceptional curve of the first kind. Hence $\tilde{S}$ is a surface of general type with $p_{g}=2, q=0, K_{S}^{2}=1$.

(ix) We may assume that $f_{3}(x, y, z)=y^{3}$. Then (4.2) becomes

$$
y^{3}+x f_{4}(1, y, z)+x^{2} f_{5}(1, y, z)=0 .
$$

If $y \mid f_{4}(1, y, z)$, then $S$ is not normal and Proposition 4.5 implies that the minimal model of $S_{0}$ is a $K 3$ surface. We assume $y+f_{4}(1, y, z)$. All the singular points of $S$ on $D_{3}$ are located on the line $L: y=0, x=0$ on $S$, which are determined by the roots of $f_{4}(1,0, z)$. Let $q \in S$ be the point $x=0, y=0, z=\zeta$, where $\zeta$ is a root of $f_{4}(1,0, z)=0$. Without loss of generality, we may assume $\zeta=0$. If $z^{2}+f_{4}(1,0, z)$ then $q$ is a rational double point. Write

$$
\begin{aligned}
& f_{4}(1, y, z)=f_{4}(1,0, z)+y g(z)+y^{2} h(y, z), \\
& f_{5}(1, y, z)=f_{5}(1,0, z)+y G(z)+y^{2} H(y, z) .
\end{aligned}
$$

Assume that $z^{2} \mid f_{4}(1,0, z)$. One of the following relations must hold:

$$
z^{2}+f_{5}(1,0, z), \quad z+g(z), \quad z+G(z),
$$

otherwise $S_{0}$ would not be normal.

If $z+g(z)$, then $q$ is a rational double point.

If $z|f(z), z| f_{5}(1,0, z)$, then the hyperplanes passing through the line $L_{0}: y=0$, $z=0$ cut out a pencil of rational or elliptic curves. In fact, for any generic hyperplane $H_{0}: \lambda y+\mu z=0, \quad H_{0} \cap S_{0}=L_{0} \cup Q_{0}$ where $Q_{0}$ is an irreducible quartic curve with a double point and an infinitely near double point. Hence $S_{0}$ is not a surface of general type. 
In the remaining discussion we always assume $z \mid g(z), z+f_{5}(1,0, z)$. If $z^{3}+$ $f_{4}(1,0, z)$, then $q$ is a rational double point $E_{6}$.

If $z^{3} \mid f_{4}(1,0, z)$, then $q$ is a minimally elliptic double point of the type $\mathrm{II}_{\mathrm{a}}$. Note that there is at most one such essential singularity. Let $\tilde{S}$ be the minimal resolution of $S$. Then $\left|K_{\tilde{S}}\right|$ is cut out by all hyperplanes in $\mathbf{P}^{3}$ passing through the line $L_{0}$ : $y=0, z=0$. Since $L_{0} \not \subset S_{0}, \tilde{S}$ is a minimal surface with $p_{g}=2, q=0, K_{\tilde{S}}^{2}=1$. Hence $S_{0}$ is the surface of type III-II as stated in the theorem.

Thus far all possible cases have been discussed.

5. Normal quintic surfaces with a double point. Suppose $S_{0}$ is a normal quintic surface with an essential double point $p$. In $\S 5.1$ we will show that if $p$ is not weakly elliptic then $S_{0}$ is not a surface of general type. Then in $\$ 5.2$ we classify all normal quintic surfaces of general type with a weakly elliptic double point as the only essential singularity. Surfaces with several singularities will be treated in a separate section.

5.1 A double point is not elliptic. The major result of this subsection is

THEOREM 5.1. Let $S_{0}$ be a normal quintic surface and let $\pi: \tilde{S} \rightarrow S_{0}$ be the minimal resolution of $S_{0}$. If $S_{0}$ has a double point $p$ which is neither rational nor weakly elliptic, then $\tilde{S}$ is not a surface of general type.

As a first step to prove the theorem, we show the following lemma. Denote the fundamental cycle of $p$ by $Z$.

LEMMA 5.2. If $\chi(Z) \leqslant-2$, then $\tilde{S}$ is not a surface of general type.

Proof. Let $x^{2}=g(y, z)$ be a representative equation of the double point $p$. If $\chi(Z) \leqslant-2$, then Theorem 3.7 implies that the zero locus of $g(y, z)$ has either an $m$-tuple point with $m \geqslant 8$ or an infinitely near $n$-tuple point with $n \geqslant 7$ at $(0,0)$. So $\chi(\tilde{S}) \leqslant \chi\left(S_{0}\right)-6=-1$ by Theorem 2.12 and (2.5). Hence $\tilde{S}$ is not a surface of general type by Theorem 2.3 .

Therefore we only need to consider the case that $\chi(Z)=-1$. This is the hard part of the theorem. Let us look at some properties of this type of double points.

Let $A$ be the exceptional set of an isolated singularity $p$. Suppose that $\chi(Z)=-1$. Unlike the weakly elliptic singularities, -1 is not necessarily the minimum value of $\chi(D)$ where $D$ is an arbitrary cycle on $A$. An example is the double point $p$ defined by $x^{2}+y^{6}+z^{12}=0$, whose dual graph is

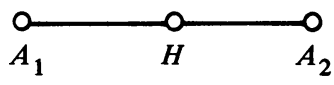

where $H$ is a nonsingular curve of genus $2, A_{1}$ and $A_{2}$ are rational curves, $H^{2}=A_{1}^{2}=A_{2}^{2}=-2$. Hence $\chi(Z)=-1$ but $\chi(Z+H)=-2$.

However we still have the following result.

Proposition 5.3. Let $p$ be an isolated singularity with $\chi(Z)=-1$. Then there is a unique cycle $H$ such that $\chi(H)=-1$ and $H \leqslant D$ for any cycle $D$ with $\chi(D)=-1$.

Proof. Let $D$ be any cycle such that $D \leqslant Z$. The restriction map $\mathcal{O}_{Z} \rightarrow \mathcal{O}_{D}$ is surjective. Since $H^{2}(M, \mathscr{F})=0$ for any coherent sheaf $\mathscr{F}$ on $M$, the map $H^{1}\left(M, \mathcal{O}_{Z}\right) \rightarrow H^{1}\left(M, \mathcal{O}_{D}\right)$ is also surjective. We know that $h^{0}\left(M, \mathcal{O}_{Z}\right)=1[12,(2.6)]$. 
Hence $\chi(D) \geqslant \chi(Z)=-1$. Here $\pi: M \rightarrow V$ is the minimal resolution of $p \in V, V$ is affine.

If $B, C$ are cycles such that $B \leqslant Z, C \leqslant Z$ and $\chi(B)=\chi(C)=-1$, then $B \wedge C \neq 0$, for otherwise $-1 \leqslant \chi(B+C)=\chi(B)+\chi(C)-B C \leqslant-2$, which is absurd. Thus

$$
\begin{aligned}
-1 & \leqslant \chi(B+C-B \wedge C) \\
& =\chi(B)+\chi(C)-\chi(B \wedge C)-(B-B \wedge C)(C-B \wedge C) \\
& \leqslant-1-1+1 .
\end{aligned}
$$

Therefore $\chi(B \wedge C)=-1$.

This shows that there is a unique cycle $H$ such that $\chi(H)=-1$ and $H \leqslant D$ for any cycle $D \leqslant Z$ with $\chi(D)=-1$.

It remains to show that $H \leqslant D$ for an arbitrary cycle $D$ with $\chi(D)=-1$. For that purpose we need a lemma.

LEMMA 5.4. Let $Z$ be the fundamental cycle of some singularity and let $D$ be a cycle such that $\chi(C) \geqslant 0$ for all $C \leqslant D \wedge Z$. Then $\chi(D) \geqslant 0$.

Proof of The Lemma. We apply induction on the degree of $D$. If $D \leqslant Z$, then the lemma is trivial. If $D \nless Z$ then $\chi(D-D \wedge Z) \geqslant 0$ by the induction hypothesis. Hence

$$
\begin{aligned}
\chi(D) & =\chi(D \wedge Z)+\chi(D-D \wedge Z)-(D \wedge Z)(D-D \wedge Z) \\
& \geqslant-(D \wedge Z)(D-D \wedge Z) \\
& =(Z-D \wedge Z)(D-D \wedge Z)-Z(D-D \wedge Z) \geqslant 0
\end{aligned}
$$

We continue the proof of the proposition. Lemma 5.4 shows that if $\chi(D)<0$ then there must be some cycle $C \leqslant D \wedge Z$ such that $\chi(C)=-1$. Hence $H \leqslant C \leqslant D$ $\wedge Z \leqslant D$.

REMARK 5.13. (1) The above proof shows that $H \leqslant D$ for all cycles $D$ such that $\chi(D)<0$.

(2) The proposition will not be used in the proof of Theorem 5.1.

Proposition 5.5. Let $p$ be a Gorenstein singularity with $\chi(Z)=-1$. Let $K^{\prime}$ be the canonical cycle. Then $K^{\prime 2} \leqslant Z^{2}-5$.

Proof. By the definition of canonical cycle, $A_{i} K^{\prime}=-A_{i} K \leqslant 0$ for all irreducible components $A_{i}$ of $A$. Hence $Z \leqslant K^{\prime}$ by the definition of the fundamental cycle. Write $K^{\prime}=Z+D$. Then $D>0$ for $p$ is not minimally elliptic. Since $Z K=-Z K^{\prime}$ $=-Z^{2}-Z D$, we have $Z D=-Z^{2}-Z K=2 \chi(Z)=-2$. Hence $K^{\prime 2}=(Z+D)^{2}$ $=Z^{2}-4+D^{2} \leqslant Z^{2}-5$ by Theorem 2 .

Proof of TheOrem 5.1. It suffices to show that $\chi(Z)=-1$ implies that $\tilde{S}$ is not of general type. We proceed by reduction to absurdity. So we suppose that $\tilde{S}$ is of general type with $\chi(Z)=-1$.

Let $S$ be a minimal model of $\tilde{S}$. Then there is a succession of blowing-ups

$$
\tilde{S}=\tilde{S}_{0} \stackrel{\sigma_{1}}{\rightarrow} \tilde{S}_{1} \stackrel{\sigma_{2}}{\rightarrow} \tilde{S}_{2} \stackrel{\sigma_{3}}{\rightarrow} \cdots \stackrel{\sigma_{k}}{\rightarrow} \tilde{S}_{k}=S
$$


Let $\tilde{Z}$ be the sum of the fundamental cycles of all essential singularities. Denote the direct image of $\tilde{Z}$ in $\tilde{S}_{i}$ by $Z_{i}$ for $0 \leqslant i \leqslant k$. Let $a_{i}, E_{i}$ be the center and the exceptional curve of $\sigma_{i}$ respectively.

Since $K_{\tilde{S}} \sim \pi^{*} H-K^{\prime}$, where $H$ is a hyperplane section on $S_{0}$ and $K^{\prime}$ is the canonical cycle, we have $K_{\tilde{S}} D \geqslant 0$ for any irreducible curve $D$ on $\tilde{S}$ which does not meet $\tilde{Z}$. Hence $a_{i} \in Z_{i}$ for all $i$. Let $r_{i}$ be the multiplicity of $E_{i}$ in $Z_{i-1}$ and let $t_{i}$ be the multiplicity of the curve $Z_{i}$ at $a_{i}$.

Claim. The inequality $t_{i}>r_{i}$ holds for $1 \leqslant i \leqslant k$.

We prove the claim by induction. Since $r_{1}=0$, the claim is true for $i=1$. For arbitrary $i$, we have

$$
t_{i}=E_{i}\left(Z_{i-1}-r_{i} E_{i}\right)=E_{i} Z_{i-1}+r_{i}
$$

We may assume that $r_{i} \neq 0$; otherwise there will be nothing to prove. Then

$$
\begin{aligned}
t_{i}-r_{i} & =E_{i} Z_{i-1}=\left(\sigma_{i-1}^{*} E_{i}\right) Z_{i-2}=E_{i, i-1} Z_{i-2}+e_{i-1} E_{i-1} Z_{i-2} \\
& =E_{i, i-2} Z_{i-3}+e_{i-2} E_{i-2} Z_{i-3}+e_{i-1} E_{i-1} Z_{i-2} \\
& =\cdots=E_{i, 1} Z_{0}+\sum_{j=1}^{i-1} e_{j} E_{j} Z_{j-1}
\end{aligned}
$$

where $E_{i, j}$ denotes the proper transform of $E_{i}$ in $\tilde{S}_{j-1}$, and

$$
e_{j}= \begin{cases}1 & \text { if } a_{j} \in E_{i, j+1}, \\ 0 & \text { if } a_{j} \notin E_{i, j+1} .\end{cases}
$$

In other words, we have the relation

$$
t_{i}-r_{i}=E_{i, 1} \tilde{Z}+\sum_{j=1}^{i-1} e_{j}\left(t_{j}-r_{j}\right) .
$$

Since $E_{i}^{2}=-1$, we have $\sum_{j=1}^{i-1} e_{j}=-E_{i, 1}^{2}-1$. Hence

$$
t_{i}-r_{i} \geqslant E_{i, 1} \tilde{Z}-E_{i, 1}^{2}-1
$$

by the induction hypothesis. The claim will be proved if we can show that

$$
E_{i, 1} \tilde{Z}-E_{i, 1}^{2}>1 \text {. }
$$

Note that $\chi\left(\mathcal{O}_{\tilde{S}}\right)=\chi\left(\mathcal{O}_{S_{0}}\right)-\Sigma h=5-\Sigma h>0$, where $\Sigma h$ is the sum of geometric genera of all essential singularities. Hence the geometric genus of $p$ is either 3 or 4 and there is at most a minimally elliptic singularity as an essential singularity other than $p$.

By our assumption that $r_{i} \neq 0$, we infer that $E_{i, 1} \leqslant Z$. If $E_{i, 1} \nless Z$, then $E_{i, 1}$ is a component of the fundamental cycle of a minimally elliptic singularity. So $E_{i, 1} \tilde{Z}-$ $E_{i, 1}^{2}=-E_{i, 1} K_{\tilde{S}}-E_{i, 1}^{2}=2$. Hence (5.2) is valid.

If $E_{i, 1} \leqslant Z$, then $E_{i, 1} \tilde{Z}=E_{i, 1} Z$; there are the following cases:

(1) $E_{i, 1} Z=0$. In this case (5.2) is trivial.

(2) $E_{i, 1} Z=-1$.

There are three possibilities:

(2a) $\operatorname{Ord}(g)=5$. Then the zero locus of $g(y, z)$ has only one infinitely near 5-tuple point and no other infinitely near $m$-tuple point with $m \geqslant 4$. Since $E_{i, 1}$ is the 
only component of $Z$ such that $E_{i, 1} Z=-1$, the canonical resolution of $p$ shows that $E_{i, 1}^{2} \leqslant-3$. Hence (5.2) is true.

(2b) $\operatorname{Ord}(g)=6$. This is the case in $\$ 3$ that $f_{n}^{-1}\left(\bar{E}_{1}\right)$ splits into two irreducible curves by Theorem 3.3. Since the zero locus of $g(y, z)$ has no infinitely near 6-tuple point at $(0,0), E_{i, 1}^{2} \leqslant-3$. Hence (5.2) is true.

(2c) $\operatorname{Ord}(g)=7$. As we have remarked, $p$ is not of type $\mathrm{II}_{\mathrm{a}}$. If $p$ is of type $\mathrm{I}$, then $2 E_{i, 1} \leqslant Z$. Hence there is some $e_{j}(1 \leqslant j \leqslant i-1)$ such that $e_{j} \geqslant 2$. Thus inequality (5.1) is strict. Since $E_{i, 1}^{2} \leqslant-2, t_{i}-r_{i}>0$. If $p$ is of type $\mathrm{II}_{\mathrm{b}}$, then one can see that the geometric genus of $p$ is at least 5 , which has already been excluded in our present discussion.

(3) $E_{i, 1} Z=-2$.

Then $p$ is a double point of type I with $\operatorname{Ord}(g)=6$. So $E_{i, 1}^{2} \leqslant-4$. Hence (5.2) is valid. Thus far we have proved the claim.

Let $K_{i}$ be the canonical divisor of $\tilde{S}_{i}$. Then

$$
Z_{i-1} K_{i-1}=\left(\sigma_{i}^{*} Z_{i}+\left(r_{i}-t_{i}\right) E_{i}\right)\left(\sigma_{i}^{*} K_{i}+E_{i}\right)=Z_{i} K_{i}+\left(t_{i}-r_{i}\right)>Z_{i} K_{i} \text {. }
$$

Meanwhile,

$$
Z_{0} K_{0}=\tilde{Z} K_{\tilde{S}}=Z K_{\tilde{S}}+(\tilde{Z}-Z) K_{\tilde{S}}=-Z^{2}+2+(\tilde{Z}-Z) K_{\tilde{S}}
$$

Since there is at most one minimally elliptic point as an essential singularity other than $p, \tilde{Z}-Z$ is either 0 or a minimally elliptic cycle. In any case (5.3) becomes $Z_{0} K_{0}=-Z^{2}+2-(\tilde{Z}-Z)^{2}$. Hence

$$
k \leqslant-Z^{2}+2-(\tilde{Z}-Z)^{2}
$$

by Lemma 2.1. On the other hand,

$$
1 \leqslant K_{k}^{2}=K_{0}^{2}+k \leqslant Z^{2}+(\tilde{Z}-Z)^{2}+k
$$

by Theorem 2.2 and Proposition 5.5 (with some trivial variation). Putting (5.4) and (5.5) together we have

$$
1-Z^{2}-(\tilde{Z}-Z)^{2} \leqslant k \leqslant 2-Z^{2}-(\tilde{Z}-Z)^{2} .
$$

Hence $0 \leqslant Z_{k} K_{k} \leqslant 1, Z_{k}^{2} \geqslant 1, K_{k}^{2} \geqslant 1$, which contradicts the Algebraic Index Theorem.

5.2 Normal quintic surfaces with a weakly elliptic double point. We state the main theorem first.

THEOREM 5.6. All normal quintic surfaces of general type with one double point as the only essential singularity are classified as follows:

Type $\mathrm{II}_{\mathrm{a}}: p$ is a minimally elliptic double point of type $\mathrm{II}_{\mathrm{a}}, \tilde{S}$ is minimal, $p_{g}(\tilde{S})=3$, $q(\tilde{S})=0, K_{\tilde{S}}^{2}=4$;

Type $\mathrm{II}_{\mathrm{a}}^{2}: p$ is a weakly elliptic double point of type $\mathrm{II}_{\mathrm{a}}$ with $h=2, \tilde{S}$ is minimal, $p_{g}(\tilde{S})=2, q(\tilde{S})=0, K_{\tilde{S}}^{2}=3$;

Type $\mathrm{II}_{\mathrm{a}}^{3}: p$ is a weakly elliptic double point of type $\mathrm{II}_{\mathrm{a}}$ with $h=3, \tilde{S}$ is minimal, $p_{g}(\tilde{S})=1, q(\tilde{S})=0, K_{\tilde{S}}^{2}=2$;

Type $\mathrm{II}_{\mathrm{b}}: p$ is a weakly elliptic double point of type $\mathrm{II}_{\mathrm{b}}$ with $h=2, \tilde{S}$ is minimal, $p_{g}(\tilde{S})=2, q(S)=0, K_{\tilde{S}}^{2}=2$;

Type I: $p$ is a minimally elliptic double point of type I, $\tilde{S}$ is minimal, $p_{g}(\tilde{S})=3$, $q(\tilde{S})=0, K_{\tilde{S}}^{2}=3$; 
Type $\mathrm{I}^{2,0}: p$ is a weakly elliptic double point of type $\mathrm{I}$ with $h=2, Z_{B_{1}}^{2}=-2, \tilde{S}$ is minimal, $p_{g}(\tilde{S})=2, q(\tilde{S})=0, K_{\tilde{S}}^{2}=1$;

Type $\left(\mathrm{I}^{2,0}\right)^{\prime}: p$ is a weakly elliptic double point of type $\mathrm{I}$ with $h=2, Z_{B_{1}}^{2}=-2, \tilde{S}$ is the blowing-up of a minimal surface $S$ with $p_{g}(S)=2, q(S)=0, K_{S}^{2}=2$;

Type $\mathrm{I}^{1,1}: p$ is a weakly elliptic double point of type $\mathrm{I}$ with $h=2, Z_{B_{1}}^{2}=-1, \tilde{S}$ is minimal, $p_{g}(\tilde{S})=2, q(\tilde{S})=0, K_{\tilde{S}}^{2}=2$;

Type $\left(\mathrm{I}^{2,1}\right)^{\prime}: p$ is a weakly elliptic double point of type $\mathrm{I}$ with $h=3, Z_{B_{1}}^{2}=-2$, $Z_{B_{2}}^{2}=-1, \tilde{S}$ is the blowing-up of a minimal surface $S, p_{g}(S)=1, q(S)=0, K_{S}^{2}=1$;

Type $\mathrm{I}^{1,2}: p$ is a weakly elliptic double point of type $\mathrm{I}$ with $h=3, Z_{B_{1}}^{2}=Z_{B_{2}}^{2}=-1$, $\tilde{S}$ is minimal, $p_{g}(\tilde{S})=1, q(\tilde{S})=0, K_{\tilde{S}}^{2}=1$.

Here $\tilde{S}$ is the minimal resolution of $S$, and $Z_{B_{1}}$ and $Z_{B_{2}}$ are defined in Definition 2.14 .

EXAMPLES. (1) The equation $x^{2}+x z^{3}+y^{3}+y^{5}+x^{5}=0$ defines a normal quintic surface $S_{0}$ with a double point $p=(0,0,0)$, which is equivalent to the double point given by $x^{2}=y^{3}+z^{6}$. Hence this is a surface of type $\mathrm{II}_{\mathrm{a}}$. Note that the fundamental cycle is nonsingular.

(2) The equation $\left(x+z^{3}\right)^{2}+\left(y-z^{2}\right)^{3}+x^{3} y^{2}+x^{5}=0$ gives a quintic surface of type $\mathrm{II}_{\mathrm{a}}^{2}$. Actually $(0,0,0)$ is equivalent to the double point given by $x^{2}+y^{3}+z^{13}$ $=0$.

The surface has also a rational double point at infinity.

(3) The equation

$$
\begin{array}{r}
{\left[\left(x+y z+z^{3}\right)^{2}+\left(y-z^{2}\right)^{3}\right]-\frac{3}{8}\left[2 x^{3}\left(x+y z+z^{3}\right)-2 x^{3} z^{3}-x^{4}\right]+\frac{1}{32} x^{5}} \\
-3\left[12 x y\left(y-z^{2}\right)^{2}-4 x\left(y-z^{2}\right)^{3}-x\left(x+2 z^{3}\right)^{2}\right]=0
\end{array}
$$

gives a quintic surface of type $\mathrm{II}_{\mathrm{a}}^{3}$. In fact, $(0,0,0)$ is a double point equivalent to $x^{2}+y^{3}+z^{18}=0$.

(4) The equation $\left(x+z^{2}\right)^{2}+z y^{4}+z x^{3}=0$ gives a quintic surface of type $\mathrm{II}_{\mathrm{b}}$.

(5) The equation $x^{2}+y^{4}+z^{4}+x^{5}=0$ gives a quintic surface of type I. The fundamental cycle is a nonsingular elliptic curve.

(6) The equation $\left(x+z^{2}\right)^{2}+y^{4}+y^{5}+x^{4}=0$ gives a quintic surface without a line through $(0,0,0)$. This is type $\mathrm{I}^{2,0}$.

(7) The equation $x^{2}+y^{4}+x z^{4}+x^{5}=0$ gives a quintic surface of type $\left(\mathrm{I}^{2,0}\right)^{\prime}$. The fundamental cycle is given by

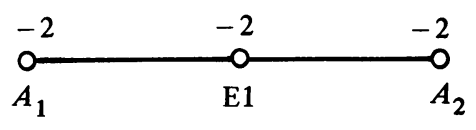

where E1 is a nonsingular elliptic curve.

(8) The equation $\left(x-z^{2}\right)^{2}+z y^{3}+x^{5}=0$ gives a quintic surface of type $I^{1,1}$. The fundamental cycle is

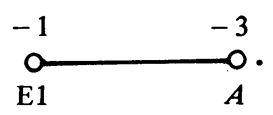


(9) Let $S_{0}$ be a quintic surface defined by the equation

$$
\left(x+a y z^{2}+z^{4}\right)^{2}+b\left(y+z^{2}\right)^{4}+c\left(y+z^{2}\right)^{3} z^{2}+x^{3} z^{2}=0,
$$

where $a=3+\sqrt{3}, b=3+2 \sqrt{3}, c=-2-2 \sqrt{3}$. The left-hand side of (5.6) is

$$
x^{2}+2 a x y z^{2}+2 x z^{4}+b y^{4}+4 b y^{2} z^{2}+c y^{3} z^{2}+x^{3} z^{2} .
$$

Hence $S_{0}$ is really a quintic surface. The double point $(0,0,0)$ is equivalent to that given by

$$
x^{2}+y^{4}+y^{3} z^{2}+z^{14}=0
$$

The dual graph of the fundamental cycle $Z$ on $\tilde{S}$ is given by

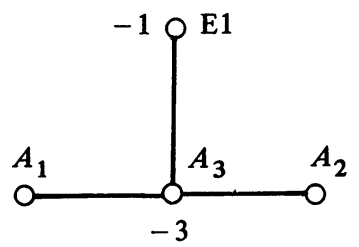

where $\mathrm{E} 1$ is a nonsingular elliptic curve. The line $L_{0}: x=0, y=0$ lies on $S_{0}$. The proper transform $\tilde{L}$ of $L_{0}$ in $\tilde{S}$ meets $A_{3}$ transversally and does not meet any of E1, $A_{1}, A_{2}$. Hence $\tilde{L} K_{\tilde{S}}=1-\tilde{L} K^{\prime}=-1$. That means that $\tilde{L}$ is an exceptional curve of the first kind. Hence $\tilde{S}$ is not a minimal surface. It can be checked that $S_{0}$ has at most rational double points besides $(0,0,0)$. Hence $S_{0}$ is of type $\left(I^{2,1}\right)^{\prime}$.

In order to prove the theorem, we need some lemmas.

LEMMA 5.7. If $p$ is a double point of type II, then $\tilde{S}$ is a minimal surface.

Proof. The proof is quite similar to that of Theorem 5.1. Let $S$ be a minimal model of $\tilde{S}$. Then there is a succession of blowing-ups

$$
\tilde{S}=\tilde{S}_{0} \stackrel{\sigma_{1}}{\rightarrow} \tilde{S}_{1} \stackrel{\sigma_{2}}{\rightarrow} \tilde{S}_{2} \rightarrow \cdots \stackrel{\sigma_{k}}{\rightarrow} \tilde{S}_{k}=S
$$

Denote the direct image of the fundamental cycle $Z$ in $\tilde{S}_{i}$ by $Z_{i}$. Let $a_{i}$, $E_{i}$ be the center and exceptional curve of $\sigma_{i}$ respectively. Then $a_{i} \in Z_{i}$ for the same reason as before. Let $r_{i}$ be the multiplicity on $E_{i}$ in $Z_{i-1}$. Let $t_{i}$ be the multiplicity of the curve $Z_{i}$ at $a_{i}$.

Claim. $t_{i} \geqslant r_{i}$ for $1 \leqslant i \leqslant k$. have

We prove the claim by induction. Obviously it is true for $i=1$. For arbitrary $i$, we

$$
t_{i}-r_{i}=E_{i, 1} Z_{0}+\sum_{j=1}^{i-1} e_{j}\left(t_{j}-r_{j}\right)
$$

where $E_{i, j}$ denotes the proper transform of $E_{i}$ in $\tilde{S}_{j-1}$, and

$$
e_{j}= \begin{cases}1 & \text { if } a_{j} \in E_{i, j+1}, \\ 0 & \text { if } a_{j} \notin E_{i, j+1} .\end{cases}
$$

Since $Z$ does not contain exceptional curves of the first kind, there must be some $j$ such that $e_{j}=1$ and $r_{j}=0$. But $-1 \leqslant E_{i, 1} Z_{0} \leqslant 0$. Hence $t_{i}-r_{i} \geqslant 0$ by the induction hypothesis. Thus the claim is proved. 
Suppose that $k \geqslant 1$. Then

$$
Z_{k} K_{k}=Z_{0} K_{0}-\sum_{i=1}^{k}\left(t_{i}-r_{i}\right)=1-\sum_{i=1}^{k}\left(t_{i}-r_{i}\right) \leqslant 0
$$

Lemma 2.6 implies that $Z_{k} K_{k}=0$. On the other hand, $Z_{k}^{2}=Z_{0}^{2}+\sum_{i=1}^{k}\left(t_{i}-r_{i}\right)^{2} \geqslant$ 0 . We get a contradiction to the Algebraic Index Theorem.

LEMMA 5.8. If $p$ is a double point of type $\mathrm{I}$, then $\tilde{S}$ is either minimal or the blowing-up of a minimal surface.

Proof. Let the chain

$$
\tilde{S}=\tilde{S}_{0} \stackrel{\sigma_{1}}{\rightarrow} \tilde{S}_{1} \stackrel{\sigma_{2}}{\rightarrow} \tilde{S}_{2} \rightarrow \cdots \stackrel{\sigma_{k}}{\rightarrow} \tilde{S}_{k}=S
$$

be defined in the same way as before. It suffices to show that $k \leqslant 1$.

Suppose that $k \geqslant 2$.

Claim. $t_{1}>r_{1}, t_{2}>r_{2}, t_{i} \geqslant r_{i}$ for $3 \leqslant i \leqslant k$.

We use the induction to prove $t_{i} \geqslant r_{i}$. If $E_{i, 1} Z_{0} \geqslant-1$, then (5.7) implies that $t_{i}-r_{i} \geqslant 0$. If $E_{i, 1} Z_{0}=-2$, then $\operatorname{Ord}(g)=4$ and $E_{i, 1}^{2}=-4$. Thus there must be at least two nonzero summands inside the summation of the right-hand side of (5.7). Therefore $t_{i} \geqslant r_{i}$.

When $i=2,(5.7)$ becomes

$$
t_{2}-r_{2}=E_{2,1} Z_{0}+t_{1}
$$

If $E_{2,1} Z_{0}=0$, then (5.8) implies that $t_{2}-r_{2}>0$ immediately. If $E_{2,1} Z_{0}=-1$, then $E_{2,1}$ is not a component of $B_{1}$ (cf. Definition 2.14). Since $\tilde{S}$ is the minimal resolution of $S_{0}, E_{1} \pi^{*} H>0$. Hence $-1=E_{1} K_{\tilde{S}}=E_{1} \pi^{*} H-E_{1} K^{\prime} \geqslant 1-E_{1} K^{\prime}$, so $E_{1} Z_{0}=$ $E_{1} K^{\prime} \geqslant 2$. Hence $t_{1} \geqslant 2$, which implies $t_{2}-r_{2}>0$. The case $E_{2,1} Z_{0}=-2$ cannot happen because $E_{2,1}^{2}=-2$. Therefore the claim is true.

By the claim we have $2=Z_{0} K_{0}>Z_{1} K_{1}>Z_{2} K_{2} \geqslant \cdots \geqslant Z_{k} K_{k} \geqslant 0$. Hence $Z_{k} K_{k}=0$. Meanwhile $Z_{k}^{2} \geqslant 0, K_{k}^{2}>0$. We get a contradiction.

Lemma 5.9. Let $p$ be a double point of type I. Assume that $Z_{B_{1}}^{2}=-1$. Then $\tilde{S}$ is minimal.

Proof. It is obvious that the linear system $\left|K_{\tilde{S}}+\sum_{i \geqslant 1} Z_{B_{i}}\right|$ is cut out by all hyperplanes in $\mathbf{P}^{3}$ passing through $p$. Hence $D\left(K_{\tilde{S}}+\sum_{i \geqslant 1} Z_{B_{i}}\right) \geqslant 0$ for any effective divisor $D$ on $\tilde{S}$ which is not a component of $Z$. Therefore if there is an exceptional curve $E$ on $\tilde{S}$ then $E\left(\sum_{i \geqslant 1} Z_{B_{i}}\right)>0$. In particular, $E Z_{B_{1}}>0$. Let $\tau$ : $\tilde{S} \rightarrow S$ be the contraction of $E$. Suppose that $\tau^{*} \tau_{*} Z_{B_{1}}=Z_{B_{1}}+r E$. Then

$$
\left(\tau_{*} Z_{B_{1}}\right)^{2}=Z_{B_{1}}^{2}+r^{2}=r^{2}-1 \geqslant 0
$$

while

$$
\left(\tau_{*} Z_{B_{1}}\right) K_{S}=Z_{B_{1}}\left(\tau^{*} K_{S}\right)=Z_{B_{1}}\left(K_{\tilde{S}}-E\right)=-r+1
$$

The intersection matrix of the divisor $\tau_{*} Z_{B_{1}}$ and $K_{S}$ becomes

$$
\left(\begin{array}{cc}
r^{2}-1 & 1-r \\
1-r & K_{S}^{2}
\end{array}\right)
$$

This is a nonnegative definite matrix. Hence we get a contradiction to the Algebraic Index Theorem. 
LEMMA 5.10 (YAU [18]). If $p$ is a weakly elliptic double point, then $Z^{2} \leqslant Z_{B_{1}}^{2} \leqslant \cdots$ $\leqslant Z_{B_{m}}^{2}<0$, where $\left\{Z_{B_{0}}=Z, Z_{B_{1}}, \ldots, Z_{B_{m}}\right\}$ is the elliptic sequence of $p$.

Proof. Let $\pi: \tilde{S} \rightarrow S_{0}$ be the minimal resolution of $p$. Then $A_{i} K_{\tilde{S}} \geqslant 0$ for any irreducible component $A_{i}$ of $Z$. As $Z \geqslant Z_{B_{1}} \geqslant \cdots \geqslant Z_{B_{m}}$, we have $K_{\tilde{S}} Z \geqslant K_{\tilde{S}} Z_{B_{1}}$ $\geqslant \cdots \geqslant K_{\tilde{S}} Z_{B_{m}}$. Since $\chi\left(Z_{B_{i}}\right)=0$ for all $i$, the Adjunction Formula implies the inequality we want.

DEFINITION 5.11 (YAU [19]). Let $p$ be a weakly elliptic singularity on a surface. If the geometric genus $h$ of $p$ is equal to the length of the elliptic sequence, then $p$ is called a maximally elliptic singularity.

Yau proved that $h$ cannot be greater than the length of the elliptic sequence if $p$ is Gorenstein. He also showed an example (due to Laufer) in which $p$ is given by $x^{2}=z\left(y^{4}+z^{6}\right), h=2$, while the length of the elliptic sequence is 3 . Actually there are more double points of this kind.

EXAMPLE 5.12. Let $p$ be a weakly elliptic double point given by $x^{2}=$ $z\left(y^{4}+z^{4 n+2}\right)(n \geqslant 1)$. Then $h=n+1$ by Theorem 2.12 . The dual graph is

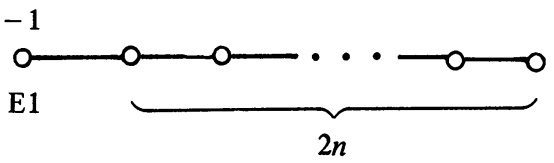

where E1 is a nonsingular elliptic curve. Hence the length of the elliptic sequence is $2 n+1$.

We will show that the above-given series of double points are essentially the only weakly elliptic double points which are not maximally elliptic.

Proposition 5.13. All weakly elliptic double points of type $\mathrm{I}$ or type $\mathrm{II}_{\mathrm{a}}$ are maximally elliptic.

Proof. If $p$ is of type $\mathrm{II}_{\mathrm{a}}$, then $p$ can be represented by $x^{2}=g(y, z)$, where $\operatorname{Ord}(g)=3$. Suppose that the locus $g(y, z)=0$ has $n$ infinitely near triple points over $(0,0)$. Then the geometric genus $h$ of $p$ is equal to $[(n+1) / 2]$ according to Theorem 2.12. The weighted dual graph is

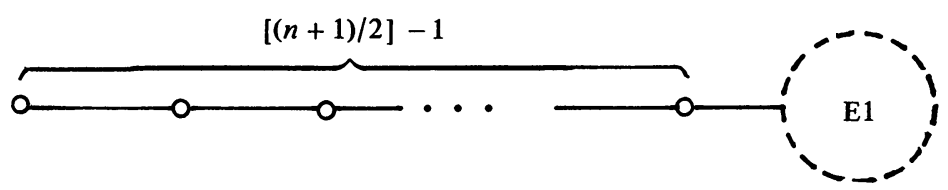

where $\mathrm{E} 1$ is the minimally elliptic cycle with $\mathrm{E} 1^{2}=-1$. It is easy to see that the length of the elliptic sequence is $[(n+1) / 2]$.

For the double point $p$ of type I, we apply induction on the geometric genus $h$ of $p$. We may assume that $p$ is not minimally elliptic. By Theorem $3.3, Z=\bar{Z}$ on $\tilde{S}$. Hence the image of $Z_{B_{1}}$ in $Y_{1}$ (for notation see §3) is a point $s$ on $E_{1}$, as $Z_{B_{1}} Z=0$. Since $4 \leqslant \operatorname{Ord}(g) \leqslant 5, p_{1}=f_{1}^{-1}(s)$ is the only double point on $X_{1}$ which is not rational. The geometric genus $h_{1}$ of $p_{1}$ is equal to $h-1$, which is equal to the length of the elliptic sequence of $p_{1}$ by the induction hypothesis. Obviously $Z_{B_{1}}$, $Z_{B_{2}}, \ldots, Z_{B_{m}}$ is the elliptic sequence of $p_{1}$ by definition. Hence the proposition is true. 
Proposition 5.14. Let $p$ be a weakly elliptic double point. The following statements are equivalent:

(i) $p$ is not maximally elliptic;

(ii) the type of $p$ is $\mathrm{II}_{\mathrm{b}}$;

(iii) $p$ can be represented by an equation $x^{2}=g(y, z)$ with $\operatorname{Ord}(g)=5$ such that the weighted dual graph is

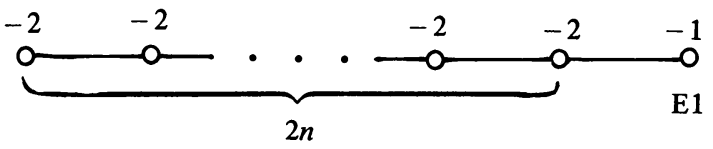

where E1 is a nonsingular elliptic curve, and the geometric genus of $p$ is equal to $n+1$.

Proof. Proposition 5.13 implies that (i) $\Rightarrow$ (ii). The part (iii) $\Rightarrow$ (i) is trivial by the definition. It remains to prove (ii) $\Rightarrow$ (iii). So we assume that $p$ is of type $\mathrm{II}_{\mathrm{b}}$. Then $\operatorname{Ord}(g)=5$. Let $Z^{\prime}$ be the redundancy cycle. Using the notation in $\S 3$, we have $Z=A_{0}+D, Z^{\prime}=A_{0}+D^{\prime}$ with $D^{\prime} \leqslant D$ and $A_{0} \nless D$. We also have $-1=A_{0} \bar{Z}=$ $A_{0}\left(2 A_{0}+D+D^{\prime}\right)=2 A_{0}^{2}+A_{0} D+A_{0} D^{\prime}$. On the other hand, by the definition of $Z^{\prime}$ we have $-1=A_{0} Z^{\prime}=A_{0}^{2}+A_{0} D^{\prime}$. These two equalities yield $A_{0}\left(D-D^{\prime}\right)=1$. Let $B_{1}$ be the branch locus of $f_{1}$. Then $E_{1}\left(B_{1}-E_{1}\right)=5$. Since $p$ is weakly elliptic, $E_{1} \cap\left(B_{1}-E_{1}\right)$ consists of at least two points. Since $\chi\left(Z^{\prime}\right)=0, p$ is not minimally elliptic. Hence there must be some point $s$ on $E_{1} \cap\left(B_{1}-E_{1}\right)$ such that

$$
\left(E_{1}, B_{1}-E_{1}\right)_{s} \geqslant 3 \text {. }
$$

Suppose $B_{1} \cap\left(B_{1}-E_{1}\right)$ consists of three points. Then $B_{1}$ will meet $B_{1}-E_{1}$ at two points transversally. That will contradict the fact that $A_{0}\left(D-D^{\prime}\right)=1$. Assume that $E_{1} \cap\left(B_{1}-E_{1}\right)=\left\{s, s^{\prime}\right\}$. Since $Z^{\prime}$ is an elliptic cycle, $\left(E_{1}, B_{1}-E_{1}\right)_{s}=3$ is impossible. Hence $\left(E_{1}, B_{1}-E_{1}\right)_{s}=4,\left(E_{1}, B_{1}-E_{1}\right)_{s^{\prime}}=1$. Since there is only one component $A_{i}$ of $Z$ such that $A_{i} Z^{\prime}>0$ and in this situation the inverse image of $s^{\prime}$ in $X^{*}$ has this property, each component of the inverse image of $s^{\prime}$ in $X^{*}$ must be a component of $Z^{\prime}$. Therefore $s$ cannot be a double or a quadruple point of $B_{1}$. If $s$ is a triple point of $B_{1}$, there must be an infinitely near triple point over it for $p$ is not minimally elliptic. Hence $h=2$ and the dual graph of the fundamental cycle is

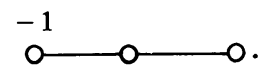

E1

If $s$ is a 5-tuple point of $B_{1}$, then I claim that $f_{1}^{-1}(s)$ is a double point of type $\mathrm{II}_{\mathrm{b}}$ on $X_{1}$. In fact, $\left(Z^{\prime}-A_{0}\right) A_{i} \leqslant 0$ for each component $A_{i}$ of $Z^{\prime}-A_{0}$, so $f_{1}^{-1}(s)$ is not of type I. Moreover, there is no infinitely near 5-tuple point over $s$. Hence $f_{1}^{-1}(s)$ cannot be of type $\mathrm{II}_{\mathrm{a}}$.

Now we can repeat the same discussion or rather use induction to finish the proof.

Proof of TheOrem 5.6. If $p$ is a double point of type $\mathrm{II}_{\mathrm{a}}$, then $S_{0}$ can only be of type $\mathrm{II}_{\mathrm{a}}$ or $\mathrm{II}_{\mathrm{a}}^{2}$ or $\mathrm{II}_{\mathrm{a}}^{3}$ because of Theorem 2.16. If $p$ is of type $\mathrm{II}_{\mathrm{b}}$, then $\tilde{S}$ is minimal by Lemma 5.7. Suppose that $h=n+1$. Then $K_{S}^{2}=5-(2 n+1)=4-2 n$. Hence $n=1$. Suppose that $p$ is of type I. Let $\left\{Z, Z_{B_{1}}, \ldots, Z_{B_{m}}\right\}$ be the elliptic sequence. If $Z_{B_{i}}^{2}=-2$ for all $i$, then $K_{S}^{2}=5-2(m+1)$. By Lemma 5.8 and Theorem 2.2, we 
have $m \leqslant 1$. If $m=0$, then $\tilde{S}$ is minimal because $\left|K_{\tilde{S}}\right|$ has no fixed component which is exceptional. If $m=1$ then $S_{0}$ is either of type $\mathrm{I}^{2,0}$ or of type $\left(\mathrm{I}^{2,0}\right)^{\prime}$ depending upon whether $\tilde{S}$ is minimal or not. If $Z_{B_{1}}^{2}=-1$, then $\tilde{S}$ is minimal by Lemma 5.9. Since $K_{S}^{2}=5-2-m>0$, we have $m \leqslant 2$. Finally, if $Z_{B_{1}}^{2}=-2$ and $Z_{B_{m}}^{2}=-1$, then $K_{\tilde{S}}^{2} \geqslant 5-4-1=0$ and the equality holds if $m=2$. In order that $\tilde{S}$ is of general type, $\tilde{S}$ must not be minimal. So this type is $\left(I^{2,1}\right)^{\prime}$. Thus far we have covered all possible cases.

REMARK. The existence of a quintic surface of type $I^{1,2}$ is still unknown.

6. Normal quintic surfaces of general type with more than one essential singularity. In this section we are going to find all the remaining quintic surfaces of general type. First we only consider surfaces with minimally elliptic double and triple points and then we take all weakly elliptic points into account.

6.1 Surfaces with minimally elliptic points. As before, we denote three types of weakly elliptic double points by $\mathrm{I}_{1} \mathrm{II}_{\mathrm{a}}$ and $\mathrm{II}_{\mathrm{b}}$. We denote a weakly elliptic triple point by III. Without special mention we will assume all essential singular points minimally elliptic. We denote a quintic surface with $m$ singular points $p_{1}, \ldots, p_{m}$ of types $t_{1}, \ldots, t_{m}$ by $\left(t_{1}, \ldots, t_{m}\right)$, e.g., $\left(\mathrm{I}, \mathrm{II}_{\mathrm{a}}, \mathrm{II}_{\mathrm{a}}\right),\left(\mathrm{II}_{\mathrm{a}}, \mathrm{III}\right)$, etc.

THEOREM 6.1. All normal quintic surfaces of general type with more than one minimally elliptics point as the only essential singularities are classified as follows:

\begin{tabular}{l|l|l|l} 
Type & $p_{g}(S)$ & $K_{S}^{2}$ & \\
\hline$(\mathrm{I}, \mathrm{I})$ & 2 & 1 & $\begin{array}{l}\text { The line passing the two double points } \\
\text { is not on the surface. } \\
\text { The line passing the two double points } \\
\text { is on the surface. }\end{array}$ \\
$(\mathrm{I}, \mathrm{I})^{\prime}$ & 2 & 2 & The line passing I,I is on the surface. \\
$\left(\mathrm{I}, \mathrm{II}_{\mathrm{a}}\right)$ & 2 & 2 & \\
$\left(\mathrm{I}, \mathrm{I}_{1}, \mathrm{II}_{\mathrm{a}}\right)^{\prime}$ & 1 & 1 & \\
$\left(\mathrm{I}, \mathrm{II}_{\mathrm{a}}, \mathrm{II}_{\mathrm{a}}\right)$ & 1 & 1 & \\
$\left(\mathrm{II}_{\mathrm{a}}, \mathrm{II}_{\mathrm{a}}\right)$ & 2 & 3 & \\
$\left(\mathrm{II}, \mathrm{II}_{\mathrm{a}}, \mathrm{II}_{\mathrm{a}}\right)$ & 1 & 2 & \\
$\left(\mathrm{II}, \mathrm{III}_{\mathrm{a}}\right)$ & 2 & 1 & The line passing the two double points is \\
$(\mathrm{I}, \mathrm{III})^{\prime}$ & 2 & 1 & on the surface.
\end{tabular}

Here the prime "" means that the minimal resolution of the quintic surface contains exactly one exceptional curve of the first kind; $S$ is a minimal model.

Proof. Since each essential singular point reduces the Euler characteristic by 1 , a quintic surface of general type can have at most four essential singular points.

Let $S_{0}$ be a quintic surface of general type which has $m_{1}$ double points of type I, $m_{\mathrm{II}_{\mathrm{a}}}$ double points of type $\mathrm{II}_{\mathrm{a}}$ and $m_{\mathrm{III}}$ triple points. Then $m_{\mathrm{I}}+m_{\mathrm{II}_{\mathrm{a}}}+m_{\mathrm{III}} \leqslant 4$. Let $\pi: \tilde{S} \rightarrow S_{0}$ be the minimal resolution of $S_{0}$. Let $\tau: \tilde{S} \rightarrow S$ be a birational morphism onto a minimal model $S$ of $\tilde{S}$.

We need some lemmas. 
LEMMA 6.2. Let $E$ be an exceptional curve of the first kind on $\tilde{S}$. Then $\pi(E)$ must pass through at least two minimally elliptic points.

Proof. If $\pi(E)$ does not pass through any essential singular points, then $E K_{\tilde{S}} \geqslant 0$. Hence $E$ cannot be an exceptional curve of the first kind. If $\pi(E)$ passes only one singular point $p_{i}$, then $K_{\tilde{S}} \sim \pi^{*} H-C_{i}-\sum_{j \neq i} C_{j}$ where $H$ is a generic hyperplane section passing through $p_{i}$ and $C_{j}$ 's are all elliptic cycles of the essential singularities. Since $E C_{j}=0$ for $j \neq i$ and $\pi^{*} H-C_{i}$ is an effective divisor not containing $E, E K_{\tilde{s}} \geqslant 0$. Hence $E$ cannot be an exceptional curve of the first kind.

LEMMA 6.3. If $m_{\mathrm{III}} \geqslant 2$, then $\tilde{S}$ is not a surface of general type.

Proof. Let $L$ be a line passing through two triple points. For a generic hyperplane $H$ passing through $L, H \cap S_{0}$ is the union of $L$ and a quartic curve $Q$ with at least two double points. Since the geometric genus of $Q$ is 0 or 1 , the projection from $L$ induces a morphism from $\tilde{S}$ onto $\mathbf{P}^{1}$, of which the generic fibre is a rational or elliptic curve.

LEMMA 6.4. If $m_{\mathrm{III}} \leqslant 1$, then $3 m_{\mathrm{I}} / 2+m_{\mathrm{II}_{\mathrm{a}}}+2 m_{\mathrm{III}} \leqslant 4$.

Proof. Let $\tilde{C}_{i}$ be an elliptic cycle on $\tilde{S}$ of an elliptic point $p_{i}$ on $S_{0}$. Let $C_{i}$ be the direct image of $\tilde{C}_{i}$ in $S$. By the Algebraic Index Theorem, $C_{i}^{2}<0$. Hence there are at most two (one, zero resp.) exceptional curves whose direct images in $S_{0}$ pass through III (I, $\mathrm{II}_{\mathrm{a}}$ resp.). Since $m_{\mathrm{III}} \leqslant 1, \pi(E)$ must pass through a double point by Lemma 6.2 for any exceptional curve $E$ of first kind. Hence $\tau\left(A_{j}\right)$ is not a point for any component $A_{j}$ of the exceptional set of $\pi$, so we have $K_{S}^{2}-K_{S}^{2} \leqslant$ $\left(m_{\mathrm{I}}+2 m_{\mathrm{III}}\right) / 2$. On the other hand, $K_{\tilde{S}}^{2}=5+\sum \tilde{C}_{i}^{2}=5-2 m_{\mathrm{I}}-m_{\mathrm{II}_{\mathrm{a}}}-3 m_{\mathrm{III}}$. The result follows immediately from the criterion $K_{S}^{2}>0$.

To prove the theorem, it suffices to consider the following three cases:

Case 1. $m_{\mathrm{I}}+m_{\mathrm{II}_{\mathrm{a}}}+m_{\mathrm{III}}=2$ and $m_{\mathrm{III}} \leqslant 1$.

The canonical system $\left|K_{\tilde{S}}\right|$ is cut out by all hyperplanes in $\mathbf{P}^{3}$ passing through the two singular points. Let $L$ be the line passing through these two points. If $L \subset S_{0}$ then the proper transform of $L$ in $\tilde{S}$ is the only exceptional curve of the first kind on $\tilde{S}$. If $L \not \subset S_{0}$, then $\tilde{S}$ is minimal. Suppose $L \subset S_{0}$ and $m_{\mathrm{II}_{\mathrm{a}}} \neq 0$. Let $\tilde{C}$ be the elliptic cycle on $\tilde{S}$ of a double point of type $\mathrm{II}_{\mathrm{a}}$. Let $C$ be the direct image of $\tilde{C}$ in $S$. Since $\tilde{C}^{2}=-1$, we have $C^{2}=0$ and $C K_{S}=0$. This contradicts the Algebraic Index Theorem. Hence $S_{0}$ can only be one of the types (I, I), (I, I)', (I, II a $),\left(\mathrm{II}_{\mathrm{a}}, \mathrm{II}_{\mathrm{a}}\right)$, (I, III)', (II ${ }_{\mathrm{a}}$, III $)$.

Case 2. $m_{\mathrm{I}}+m_{\mathrm{II}_{\mathrm{a}}}+m_{\mathrm{III}}=3$ and $m_{\mathrm{III}} \leqslant 1$.

If $m_{\mathrm{III}}=1$, then $m_{\mathrm{I}}=0, m_{\mathrm{II}}=2$ by Lemma 6.4 . Hence $\tilde{S}$ is minimal by Lemma 6.2. But $K_{\tilde{S}}^{2}=0$ contradicts the assumption that $\tilde{S}$ is of general type. Hence there are no triple points on $S_{0}$.

These three double points cannot be on a line. Otherwise the line $L$ passing through them would be on $S_{0}$. Then the proper transform $\tilde{L}$ of $L$ on $\tilde{S}$ would satisfy $\tilde{L} K_{\tilde{S}} \leqslant-2$, which is impossible. 
The canonical system $\left|K_{\tilde{S}}\right|$ is cut out by the plane $H$ passing through these three double points. An exceptional curve of the first kind is the proper transform of either a line passing through two double points or a degree $r(2 \leqslant r \leqslant 5)$ rational curve on $H$ passing through the double points $r+1$ times. By the Algebraic Index Theorem, it is easy to see that there is at most one exceptional curve of the first kind on $S$, and $S$ is minimal if $m_{\mathrm{II}_{\mathrm{a}}} \geqslant 2$. On the other hand,

$$
K_{\tilde{S}}^{2}=5-2 m_{\mathrm{I}}-m_{\mathrm{II}} \begin{cases}>0 & \text { if } \tilde{S} \text { is minimal, } \\ \geqslant 0 & \text { if } \tilde{S} \text { is not minimal }\end{cases}
$$

by Theorem 2.7. Hence $S_{0}$ can only be one of the types $\left(\mathrm{I}, \mathrm{I}, \mathrm{II}_{\mathrm{a}}\right)^{\prime},\left(\mathrm{I}, \mathrm{II}_{\mathrm{a}}, \mathrm{II}_{\mathrm{a}}\right)$, $\left(\right.$ II $\left._{\mathrm{a}}, \mathrm{II}_{\mathrm{a}}, \mathrm{II}_{\mathrm{a}}\right)$.

Case 3. $m_{\mathrm{I}}+m_{\mathrm{II}}+m_{\mathrm{III}}=4$ and $m_{\mathrm{III}} \leqslant 1$.

The only possibility is $m_{1}=m_{\mathrm{III}}=0, m_{\mathrm{II}_{\mathrm{a}}}=4$ in terms of Lemma 6.4. But this surface does not exist by Theorem 2.9.

Therefore the list is complete. It is easy to check the values $p_{g}$ and $K_{S}^{2}$.

THEOREM 6.5. All the surfaces in Theorem 6.1 are regular.

Proof. The irregularity of $S$ is $q=\chi(S)-1-p_{g}=\chi(\tilde{S})-1-p_{g}$. By (2.5),

$$
\chi(\tilde{S})=\chi\left(S_{0}\right)-\left(m_{\mathrm{I}}+m_{\mathrm{II}_{\mathrm{a}}}+m_{\mathrm{III}}\right)=5-\left(m_{\mathrm{I}}+m_{\mathrm{II}_{\mathrm{a}}}+m_{\mathrm{III}}\right) .
$$

Theorem 6.1 shows that $p_{g}=4-\left(m_{\mathrm{I}}+m_{\mathrm{II}_{\mathrm{a}}}+m_{\mathrm{III}}\right)$. Hence $q=0$.

EXAMPLES. Here we give various examples to show the existence of the surfaces in Theorem 6.1. We only give the details of the verification in the first example. All the other examples can be verified by the same method. All equations are given in terms of three variables $x, y, z$. The remaining letters $a, b, c, d, \ldots$ are generic parameters.

(1) The equation $(x+z)^{2}+(x+z) z^{2}+b y^{4}+c y^{4} z+d x^{4} z=0$ gives a quintic surface $S_{0}$ of type $(\mathrm{I}, \mathrm{I})$. We verify it by the following procedure:

(i) At $p_{1}=(0,0,0), S_{0}$ has a double point which is equivalent to that given by $x^{2}+y^{4}+z^{4}=0$. This is a minimally elliptic double point of type $\mathrm{I}$ with a nonsingular elliptic curve as fundamental cycle.

(ii) At $p_{2}=(0,0, \infty)$, the equation of $S_{0}$ becomes

$$
t^{3}(x+1)^{2}+t^{2}(x+1)+b t y^{4}+c y^{4}+d x^{4}=0,
$$

which has a double point equivalent to $t^{2}+y^{4}+x^{4}=0$.

(iii) The line passing through $p_{1}$ and $p_{2}$ is not on $S_{0}$.

(iv) The surface $S_{0}$ is smooth at all other points. Actually, if we take $b=1$, $c=d=0$, then the Jacobian condition for a singular point on $S_{0}$ is

$$
\left\{\begin{array}{l}
(x+z)^{2}+(x+z) z^{2}+y^{4}=0, \quad 2(x+z)+z^{2}=0 \\
4 y^{3}=0, \quad 2(x+z)+z^{2}+2(x+z) z=0
\end{array}\right.
$$

The only solution of (6.1) is $x=0, y=0, z=0$. By Bertini's Theorem, $S_{0}$ is smooth in $\mathbf{C}^{3} \backslash\{(0,0,0)\}$. Using a similar method one can check that $S_{0}$ is smooth at all points except $p_{1}$ and $p_{2}$.

(2) The equation $a x^{2}+b x^{2} z^{3}+c x z^{2}+d y^{3} z+e y^{5}+f x^{5}=0$ gives a quintic surface $S_{0}$ of type $(\mathrm{I}, \mathrm{I})^{\prime}$. The two minimally elliptic double points are $p_{1}=(0,0,0)$ and $p_{2}=(0,0, \infty)$. The line $L: x=0, y=0$ passing through $p_{1}$ and $p_{2}$ is on $S_{0}$. 
(3) The equation $a x^{2}+b z^{3}+c x y^{3}+d x^{4} z+e y^{4} z=0$ gives a quintic surface of type $\left(\mathrm{I}_{1} \mathrm{II}_{\mathrm{a}}\right)$. The point $(0,0,0)$ is a minimally elliptic double point of type $\mathrm{II}_{\mathrm{a}}$ while $(0,0, \infty)$ is of type $I$.

(4) The equation $a x^{2}+b z^{3}+c x y^{3}+d y^{3} z^{2}+e x^{3} z=0$ gives a quintic surface $S_{0}$ of type $\left(\mathrm{II}_{\mathrm{a}}, \mathrm{II}_{\mathrm{a}}\right)$. The two minimally elliptic double points are $(0,0,0)$ and $(0,0, \infty)$. The surface $S_{0}$ has also two rational double points $(0, \infty, 0)$ and $(\infty, 0,0)$.

(5) The equation $a x^{2}+b y^{3}+c x z^{3}+d x^{2} y^{3}+e y^{5}=0$ gives a quintic surface of type $\left(\mathrm{II}_{\mathrm{a}}, \mathrm{III}\right)$. The point $(0,0,0)$ is a minimally elliptic double point of type $\mathrm{II}_{\mathrm{a}}$ while $(\infty, 0,0)$ is a minimally elliptic triple point.

(6) The equation $a x^{2}+b x z^{2}+c y^{4}+d y^{3} z^{2}+e x^{5}=0$ gives a quintic surface $S_{0}$ of type (I, III)'. The point $(0,0,0)$ is a minimally elliptic double point of type I, while $(0,0, \infty)$ is a minimally elliptic triple point. The line $L: x=0, y=0$ lies on $S_{0}$.

6.2 All the remaining cases. As before we denote an elliptic double point of type I by $\mathrm{I}^{k_{1}, k_{2}}$, an elliptic double point of type $\mathrm{II}_{\mathrm{a}}$ by $\mathrm{II}_{\mathrm{a}}^{k}$, an elliptic double point of type II $_{b}$ with geometric genus 2 (cf. §5.2) by II $_{b}$.

THEOREM 6.6. All quintic surfaces of general type with more than one essential singularity, among which at least one has geometric genus greater than one, are listed as follows:

\begin{tabular}{l|l|l} 
Type & $p_{g}(S)$ & $K_{S}^{2}$ \\
\hline$\left(\mathrm{I}^{2,0}, \mathrm{II}_{\mathrm{a}}\right)^{\prime}$ & 1 & 1 \\
$\left(\mathrm{I}^{1,1}, \mathrm{I}^{\prime}\right.$ & 1 & 1 \\
$\left(\mathrm{I}^{1,1}, \mathrm{II}_{\mathrm{a}}\right)$ & 1 & 1 \\
$\left(\mathrm{I}, \mathrm{II}_{\mathrm{a}}^{2}\right)$ & 1 & 1 \\
$\left(\mathrm{II}_{\mathrm{a}}^{2}, \mathrm{II}_{\mathrm{a}}\right)$ & 1 & 2 \\
$\left(\mathrm{II}_{\mathrm{a}}, \mathrm{II}_{\mathrm{b}}\right)$ & 1 & 1
\end{tabular}

Proof. Let $S_{0}$ be a quintic surface of general type. We know that $S_{0}$ has at most one triple point and this triple point must be minimally elliptic. Let $m_{\mathrm{I}^{k_{1}}, k_{2}}$ be the number of double points of type $\mathrm{I}^{k_{1}, k_{2}}$, let $m_{\mathrm{II}_{\mathrm{a}}^{k}}$ be the number of double points of type $\mathrm{II}_{\mathrm{a}}^{k}$, let $m_{\mathrm{II}_{\mathrm{b}}}$ be the number of double points of type $\mathrm{II}_{\mathrm{b}}$ and let $m_{\mathrm{III}}$ be the number of minimally elliptic triple points on $S_{0}$. Similar to Lemma 6.4, we have

LEMMA 6.7.

$$
\frac{1}{2}\left(\sum_{k_{1}, k_{2}} m_{\mathrm{I}^{k_{1}, k_{2}}}\left(3 k_{1}+2 k_{2}\right)\right)+\sum_{k} m_{\mathrm{II}^{k}} k+3 m_{\mathrm{II}_{\mathrm{b}}}+2 m_{\mathrm{III}} \leqslant 4 .
$$

Proof. Let $\tilde{C}_{i}$ be an elliptic cycle on $\tilde{S}$ of an essential singularity $p_{i}$ on $S_{0}$. Let $C_{i}$ be the direct image of $\tilde{C}_{i}$ in $S$. By the Algebraic Index Theorem, $C_{i}^{2}<0$. Hence there are at most two (one, zero, zero resp.) exceptional curves passing through III $\left(\mathrm{I}, \mathrm{II}_{\mathrm{a}}, \mathrm{II}_{\mathrm{b}}\right.$ resp.). On the other hand, Lemma 6.2 is still true if we take any elliptic point with geometric genus $h$ as $h$ minimally elliptic points concentrating at a single point. Hence

$$
K_{S}^{2}-K_{\tilde{S}}^{2} \leqslant \frac{1}{2}\left(\sum_{k_{1}, k_{2}} m_{\mathrm{I}^{k_{1}, k_{2}}} k_{1}+2 m_{\mathrm{III}}\right)
$$


Meanwhile, we have

$$
K_{\tilde{S}}^{2}=5-\sum_{k_{1}, k_{2}} m_{\mathrm{I}^{k_{1} \cdot k_{2}}}\left(2 k_{1}+k_{2}\right)-\sum_{k} m_{\mathrm{II}^{k}} k-3 m_{\mathrm{II}_{\mathrm{b}}}-3 m_{\mathrm{III}}
$$

Since $K_{S}^{2}>0$, the result follows immediately.

We continue the proof of the theorem. By our assumption, either there is some $\mathrm{I}^{k_{1}, k_{2}}$ with $k_{1}+k_{2} \geqslant 2$ or there is some $\mathrm{II}_{\mathrm{a}}^{k}$ with $k \geqslant 2$ or there is some $\mathrm{II}_{\mathrm{b}}$.

Case 1. $m_{\mathrm{II}_{\mathrm{b}}} \neq 0$.

Then $m_{\mathrm{II}_{\mathrm{b}}}$ must be 1 . It is clear by Lemma 6.7 that $m_{\mathrm{III}}=0, m_{\mathrm{I}^{k_{1}} \cdot k_{2}}=0$ for all $k_{1}>0, m_{\mathrm{II}^{k}}=0$ for all $k>1$ and $m_{\mathrm{II}_{2}^{\prime}}=1$.

Case 2. $m_{\mathrm{II}}=0$ and $m_{\mathrm{III}}=1$.

Then $S_{0}$ must be either $\left(\mathrm{I}^{1,1}, \mathrm{III}\right)^{\prime \prime}$ or $\left(\mathrm{II}_{\mathrm{a}}^{2}, \mathrm{III}\right)^{\prime}$ by Lemma 6.7. But this cannot happen by the Algebraic Index Theorem.

Case 3. $m_{\mathrm{II}_{\mathrm{b}}}=m_{\mathrm{III}}=0$.

If there is some $\mathrm{I}^{k_{1}, k_{2}}$ with $k_{1} \geqslant 2$, then $k_{1}=2, k_{2}=0$ and $S_{0}$ must be $\left(\mathrm{I}^{2,0}, \mathrm{II}_{\mathrm{a}}\right)^{\prime}$.

If there is some $\mathrm{I}^{1,1}$, then $m_{\mathrm{I}^{1.1}}=1$. Lemma 6.7 implies that $3 m_{\mathrm{I}^{1.0}} / 2+\sum_{k} m_{\mathrm{II}^{k}} k$ $\leqslant 2$. Hence either $m_{\mathrm{I}^{1.0}}=1, m_{\mathrm{II}_{\mathrm{a}}^{k}}=0$ for all $k$, or $m_{\mathrm{I}^{1,0}}=0$. In the former case, $S_{0}$ is $\left(\mathrm{I}^{1,1}, \mathrm{I}\right)^{\prime}$. In the latter case, $S_{0}$ will be one of $\left(\mathrm{I}^{1,1}, \mathrm{II}_{\mathrm{a}}\right),\left(\mathrm{I}^{1,1}, \mathrm{II}_{\mathrm{a}}^{2}\right)^{\prime}$ and $\left(\mathrm{I}^{1,1}, \mathrm{II}_{\mathrm{a}}, \mathrm{II}_{\mathrm{a}}\right)^{\prime}$. But neither $\left(\mathrm{I}^{1,1}, \mathrm{II}_{\mathrm{a}}\right)^{\prime}$ nor $\left(\mathrm{I}^{1,1}, \mathrm{II}_{\mathrm{a}}, \mathrm{II}_{\mathrm{a}}\right)^{\prime}$ exists by the Algebraic Index Theorem.

If all the elliptic double points of type I are minimally elliptic, then there is some $\mathrm{II}_{\mathrm{a}}^{k}$ with $k \geqslant 2$. Lemma 6.7 shows that all possible $S_{0}$ 's are $\left(\mathrm{I}, \mathrm{II}_{\mathrm{a}}^{2}\right),\left(\mathrm{II}_{\mathrm{a}}^{2}, \mathrm{II}_{\mathrm{a}}\right)$, $\left(\mathrm{II}_{\mathrm{a}}^{2}, \mathrm{II}_{\mathrm{a}}^{2}\right),\left(\mathrm{II}_{\mathrm{a}}^{2}, \mathrm{II}_{\mathrm{a}}, \mathrm{II}_{\mathrm{a}}\right),\left(\mathrm{II}_{\mathrm{a}}^{3}, \mathrm{II}_{\mathrm{a}}\right)$. But the last three surfaces have geometric genus 0 , which do not exist by Theorem 2.9.

THEOREM 6.8. All the surfaces in Theorem 6.6 are regular.

Proof. By (2.6),

$$
\chi(\tilde{S})=5-\left(\sum_{k_{1}, k_{2}} m_{\mathrm{I}^{k_{1} \cdot k_{2}}}\left(k_{1}+k_{2}\right)+\sum_{k} m_{\mathrm{II}_{\mathrm{a}}^{k}} k+m_{\mathrm{II}_{\mathrm{b}}}+m_{\mathrm{III}}\right),
$$

which is equal to 2 for all six types of surfaces in Theorem 6.6. Hence $q=1+p_{g}-$ $\chi(\tilde{S})=0$.

EXAmples. (1) The equation $a x^{2}+b y^{4}+c x z^{4}+d x^{3}+e x^{2} z^{3}+f x y^{3}=0$ gives a quintic surface of type $\left(I^{2,0}, I_{a}\right)^{\prime}$. The double point $(0,0,0)$ is equivalent to the double point given by $x^{2}+y^{4}+z^{8}=0$, while the double point $(\infty, 0,0)$ is equivalent to that given by $x^{2}+y^{3}+z^{6}=0$. The line $L_{0}: x=0, y=0$ lies on $S_{0}$.

(2) The equation $\left(x+z^{2}\right)^{2}+\left(x+z^{2}\right) z^{5}-2\left(x+z^{2}\right)^{2} z^{3}+\left(x+z^{2}\right)^{3} z+z y^{3}+$ $x y^{3}=0$ gives a quintic surface of type $\left(\mathrm{I}^{1,1}, \mathrm{II}_{\mathrm{a}}\right)$. The double point $(0,0,0)$ is equivalent to that given by $x^{2}+y^{3} z+z^{10}=0$, while the double point $(\infty, 0,0)$ is equivalent to that given by $t^{2}+z^{3}+y^{6}=0$.

(3) The equation $a\left[\left(x+z^{3}\right)^{2}+\left(y-z^{2}\right)^{3}\right]+b x^{3} y z+c x^{5}=0$ gives a quintic surface of type $\left(\mathrm{I}, \mathrm{II}_{\mathrm{a}}^{2}\right)$. The double point $(0,0,0)$ is equivalent to that given by $x^{2}+y^{3}+z^{12}=0$, while the double point $(0, \infty, 0)$ is equivalent to that given by $t^{2}+z^{4}+x^{3} z=0$. 
(4) The equation

$$
\begin{array}{r}
\left(x+z^{2}\right)^{2}+\left(x+z^{2}\right)^{3}-3\left(x+z^{2}\right)^{2} z^{2}+2\left(x+z^{2}\right) z^{4}+y^{4} z \\
+2 x^{3} z+x^{3} z^{2}+x y^{3} z+\left(x+z^{2}\right) y^{3}=0
\end{array}
$$

gives a quintic surface of type $\left(\mathrm{II}_{\mathrm{a}}, \mathrm{II}_{\mathrm{b}}\right)$. The double point $(0,0,0)$ is equivalent to that given by $x^{2}+y^{4} z+z^{7}=0$, while the double point $(\infty, 0,0)$ is equivalent to that given by $z^{2}+t^{3}+y^{6}=0$.

7. Quintic surfaces with singular curves. Let $S_{0}$ be a quintic surface and let $\pi$ : $\tilde{S} \rightarrow S_{0}$ be the minimal resolution of $S_{0}$. In this section we show the following theorem.

THEOREM 7.1. If $S_{0}$ is a quintic surface of general type, then it must be normal.

The theorem can be proved by the following sequence of lemmas.

LEMMA 7.2. If the singular locus of a quintic surface $S_{0}$ contains a singular curve with multiplicity greater than 2 , then the minimal resolution $\tilde{S}$ of $S_{0}$ is a ruled surface.

Proof. Let $C$ be a $m$-tuple curve on $S_{0}$ with $m \geqslant 3$.

If $C$ is not a line, take a generic line $L$ in $\mathbf{P}^{3}$. The line $L$ gives rise to a projection $p: S_{0} \rightarrow \mathbf{P}^{1}$. A generic fibre of $p$ is the hyperplane section $\mathrm{D}$ of $S_{0}$ by a generic plane $H$ passing through $L$, which is a quintic curve with $n m$-tuple points where $n$ is the degree of $C$. The geometric genus of $D$ is $6-n m(m-1) / 2$. Since $m \geqslant 3$, $n \geqslant 2$ by our assumption, we have $n=2$ and $m=3$. Hence $D$ is a rational curve. Hence $\tilde{S}$ is a ruled surface.

If $C$ is a line, let $p: S_{0} \rightarrow \mathbf{P}^{1}$ be the projection from $C$. For a generic plane $H$ passing through $L, H \cap S_{0}$ is the union of $C$ and a rational curve. Hence $\tilde{S}$ is still a ruled surface.

Lemma 7.3. Suppose that the quintic surface $S_{0}$ contains a double line L. Then $\tilde{S}$ is not a surface of general type.

Proof. Let $p: S_{0} \rightarrow \mathbf{P}^{1}$ be the projection from $L$. For a generic plane $H$ passing through $L, H \cap S_{0}$ is the union of $L$ and a singular rational curve or a nonsingular elliptic curve depending on whether $S_{0}$ contains other multiple curves or not. This implies that $\tilde{S}$ is a fibration of rational or elliptic curves.

LEMMA 7.4. If the singular locus of a quintic surface $S_{0}$ contains a curve of degree greater than 4 , then the minimal resolution $\tilde{S}$ of $S_{0}$ is not a surface of general type.

Proof. The projection from a generic line in $\mathbf{P}^{3}$ will give $\tilde{S}$ a structure of fibration of elliptic or rational curves.

LEMMA 7.5. If the singular locus of a quintic surface $S_{0}$ contains a conic $Q$, then $\tilde{S}$ is not a surface of general type.

Proof. Let $\pi: T \rightarrow \mathbf{P}^{3}$ be the blowing-up of $\mathbf{P}^{3}$ with center at $Q$. Let $E$ be the exceptional divisor. Then $K_{T} \sim \pi^{*} K_{\mathbf{P}^{3}}+E$. Let $S^{\prime}$ be the proper transform of $S_{0}$. 
The adjoint system $\left|K_{T}+S^{\prime}\right|$ of the surface $S^{\prime}$ is $\left|\pi^{*} H-E\right|$, where $H$ is a hyperplane in $\mathbf{P}^{3}$. We may take $H$ to be the plane on which $Q$ is located. Then $H \cap S_{0}=Q \cup L$, where $L$ is a line. Let $L^{\prime}$ be the proper transform of $L$ in $S^{\prime}$. Then $L^{\prime}$ is cut out by the adjoint system $\left|\pi^{*} H-E\right|$. Let $\tilde{S}$ be the minimal resolution of $S^{\prime}$. The canonical divisor $K_{\tilde{S}}$ is linearly equivalent to $\tilde{L}-D$, where $\tilde{L}$ is the proper transform of $L^{\prime}$ and $D$ is some effective divisor. The adjunction formula implies $-2=\tilde{L}^{2}+\tilde{L} K_{\tilde{S}}=2 \tilde{L} K_{\tilde{S}}+\tilde{L} D$. Since $\tilde{L}$ is not a component of $D$, $\tilde{L} D \geqslant 0$. Hence $\tilde{L} K_{\tilde{S}}<0$. Suppose that $\tilde{S}$ is a surface of general type. Then $\tilde{L}$ is an exceptional curve of the first kind, which implies that the minimal model of $\tilde{S}$ is either a ruled surface or a $K 3$ surface. This leads to a contradiction.

LEMMA 7.6. If the singular locus of a quintic surface $S_{0}$ contains a curve $C$ of degree 3 , then $\tilde{S}$ is not a surface of general type.

Proof. By Lemmas 7.2, 7.3 and 7.5 we may assume that $C$ is irreducible. It is clear that $C$ is not a plane curve, for otherwise the plane where $C$ is located would be a component of $S_{0}$. Hence $C$ is a twisted cubic curve. Let $\pi: T \rightarrow \mathbf{P}^{3}$ be the blowing-up of $\mathbf{P}^{3}$ at $C$, and let $E$ be the exceptional divisor. Obviously the linear system $\left|2\left(\pi^{*} H-E\right)\right|$ on $T$ is empty, where $H$ is a hyperplane in $\mathbf{P}^{3}$. Hence $\left|2 K_{\tilde{S}}\right|$ is empty, where $\tilde{S}$ is the minimal resolution of the proper transform of $S_{0}$ in $T$. Therefore $\tilde{S}$ is not a surface of general type by the criterion given in Theorem 2.7.

So far we have proved Theorem 7.1 completely.

8. Families of quintic surfaces of general type. Let Hilb denote the Hilbert scheme of all quintic surfaces in $\mathbf{P}^{3}$. It is well known that Hilb $\cong P^{N}$ where $N=\left(\begin{array}{c}5+3 \\ 3\end{array}\right)-1$ $=55$. Let $X=\left\{(f, p) \in\right.$ Hilb $\left.\times \mathbf{P}^{3} \mid p \in f\right\}$. Let $\tau: X \rightarrow$ Hilb be the restriction on $X$ of the first projection from Hilb $\times \mathbf{P}^{3}$ onto Hilb.

The aim of the remaining sections is to determine families of quintic surfaces of general type. We denote the subset of all quintic surfaces of type $T$ in Hilb by $\operatorname{Hilb}\{T\}$. Generally, $\operatorname{Hilb}\{T\}$ is a quasivariety for all types $T$ that we have studied. Denote by $p_{g}(T)$ and $K^{2}(T)$ the geometric invariants $p_{g}$ and $K^{2}$ of any quintic surface of type $T$. For fixed positive integers $m$ and $n$, let

$$
V_{m, n}=\bigcup_{\substack{p_{g}(T)=m \\ K^{2}(T)=n}} \text { Hilb } T .
$$

In other words, $V_{m, n}$ is the set of all quintic surfaces of general type with geometric invariants $p_{g}=m, q=0, K^{2}=n$.

EXAMPLE 8.1. The set of all quintic surfaces without essential singularities is exactly $V_{4.5}$. This is an open subset of Hilb by Bertini's Theorem. The dimension of $V_{4,5}$ is the same as that of Hilb, which is equal to 55 .

For other $V_{m, n}$ the following table shows all possible types of surfaces it may contain. 
TABLE 8.1

\begin{tabular}{l|l} 
& \multicolumn{1}{|c}{ types } \\
\hline$V_{3,2}$ & III \\
$V_{2,1}$ & \left.${\text { III-I' }, \mathrm{III} \mathrm{II}_{\mathrm{a}}, \mathrm{I}^{2,0},(\mathrm{I}, \mathrm{I}),(\mathrm{II}}_{\mathrm{a}}, \mathrm{III}\right),(\mathrm{I}, \mathrm{III})^{\prime}$ \\
$V_{3,4}$ & $\mathrm{II}_{\mathrm{a}}$ \\
$V_{2,3}$ & $\mathrm{II}_{\mathrm{a}}^{2},\left(\mathrm{II}_{\mathrm{a}}, \mathrm{II}_{\mathrm{a}}\right)$ \\
$V_{1,2}$ & $\mathrm{II}_{\mathrm{a}}^{3},\left(\mathrm{II}_{\mathrm{a}}, \mathrm{II}_{\mathrm{a}}, \mathrm{II}_{\mathrm{a}}\right),\left(\mathrm{II}_{\mathrm{a}}^{2}, \mathrm{II}_{\mathrm{a}}\right)$ \\
$V_{2,2}$ & $\mathrm{II}_{\mathrm{b}},\left(\mathrm{I}^{2,0}\right)^{\prime}, \mathrm{I}^{1,1},(\mathrm{I}, \mathrm{I})^{\prime},\left(\mathrm{I}_{1} \mathrm{II}_{\mathrm{a}}\right)$ \\
$V_{3,3}$ & $\mathrm{II}$ \\
$V_{1,1}$ & $\left(\mathrm{I}^{2,1}\right)^{\prime}, \mathrm{I}^{1,2},\left(\mathrm{I}_{1}, \mathrm{II}_{\mathrm{a}}\right)^{\prime},\left(\mathrm{I}_{1}, \mathrm{II}_{\mathrm{a}}, \mathrm{II}_{\mathrm{a}}\right),\left(\mathrm{I}^{2,0}, \mathrm{II}_{\mathrm{a}}\right)^{\prime}$, \\
& $\left(\mathrm{I}^{1,1}, \mathrm{I}^{\prime},\left(\mathrm{I}^{1,1}, \mathrm{II}_{\mathrm{a}}\right),\left(\mathrm{I}, \mathrm{II}_{\mathrm{a}}^{2}\right),\left(\mathrm{II}_{\mathrm{a}}, \mathrm{II}_{\mathrm{b}}\right)\right.$
\end{tabular}

9. Families of quintic surfaces of general type with a triple point. In this section we study $\operatorname{Hilb}\{T\}$ with $T=$ III, III-I', III-II, , (II, , III) and (I, III)'.

9.1 Representations as double covers. Let $S_{0}$ be a normal quintic surface of general type with a triple point $p$. Let $\pi_{0}$ be the projection of $\mathbf{P}^{3}$ from $p$ onto the plane $\mathbf{P}^{2}$. This map $\pi_{0}$ induces a rational map from $S_{0}$ to $\mathbf{P}^{2}$, which we still denote by $\pi_{0}$. Let $\sigma: T \rightarrow \mathbf{P}^{3}$ be the blowing-up of $\mathbf{P}^{3}$ at $p$. The exceptional divisor $E=\sigma^{-1}(p)$ is isomorphic to $\mathbf{P}^{2}$. It is clear that $T$ is a $P^{1}$-bundle over $\mathbf{P}^{2}$ with the following commutative diagram:

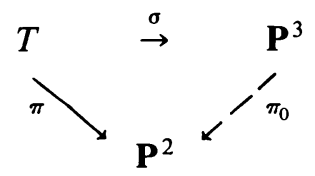

Let $S$ be the proper transform of $S_{0}$ in $T$. Then $\pi$ induces a generically finite morphism $\varphi: S \rightarrow \mathbf{P}^{2}$ of degree 2 .

LEMMA 9.1. The branch locus $B$ of the double cover $\varphi$ is a curve of degree 8 without multiple components.

Proof. Write the defining equation of $S_{0}$ as $f(x, y, z)=f_{3}(x, y, z)+f_{4}(x, y, z)$ $+f_{5}(x, y, z)=0$. The map $\pi_{0}$ is ramified at all points $(t x, t y, t z)$ such that the equation

$$
f(t x, t y, t z) / t^{3}=0
$$

in $t$ has a multiple root. The left-hand side of (9.2) is $t^{2} f_{3}(x, y, z)+t f_{4}(x, y, z)+$ $f_{5}(x, y, z)$. Let us regard $x, y, z$ as the homogeneous coordinates of $\mathbf{P}^{2}$. Then the branch locus $B$ is the curve $f_{4}^{2}(x, y, z)-4 f_{3}(x, y, z) f_{5}(x, y, z)=0$. This shows that $B$ has degree 8 . Since $S_{0}$ is normal, $B$ has no multiple components. 
LeMmA 9.2. (1) If $S_{0}$ is of type III, then $B$ has no m-tuple points with $m \geqslant 4$ or infinitely near $n$-tuple points with $n \geqslant 3$.

(2) If $S_{0}$ is of type (II $\left.\mathrm{I}_{\mathrm{a}}, \mathrm{III}\right)$, then $B$ has an infinitely near triple point away from $\varphi(C)$, where $C=E \cap S$.

(3) If $S_{0}$ is one of the types III-I', III-II ${ }_{\mathrm{a}}$ or (I, III)', then $B$ has an infinitely near triple point on $\varphi(C)$.

Proof. (1) Since $S$ has no essential singularities, the statement comes from the corollary to Theorem 2.12 .

(2) Since the line passing the triple point and the minimally elliptic double point is not on $S_{0}$, the image $w$ of the elliptic double point under $\varphi$ is not contained in $\varphi(C)$. Since that double point is of type $\mathrm{II}_{\mathrm{a}}, B$ has an infinitely near triple point at $w$.

(3) If $S_{0}$ is of type III-II ${ }_{\mathrm{a}}$, then $S$ has a minimally elliptic double point $q$ of type II $_{\mathrm{a}}$ on $C$. Hence $B$ has an infinitely near triple point at $w=\varphi(q) \in \varphi(C)$. If $S_{0}$ is of type (I, III)', then the line $L_{0}$ passing through the triple point and the double point $q$ of type I is contained in $S_{0}$. Hence $w=\varphi(q)$ is contained in $\varphi(C)$. Since the inverse image of $w$ in the canonical resolution of the double cover over $\mathbf{P}^{2}$ with branch locus $B$ contains an exceptional curve of the first kind, $B$ must have an infinitely near triple point at $w$. If $S_{0}$ is of type III-I', then there is a line $L_{0} \subset S_{0}$ such that the proper transform $L$ of $L_{0}$ in $S$ passes through the minimally elliptic double point $q$ of $S$ on $C$. Hence $w=\varphi(L)=\varphi(q)$ is a point on $\varphi(C)$ and $B$ has an infinitely near triple point at $w$.

Lemma 9.3. Let $x, y, z$ be the homogeneous coordinates of $\mathbf{P}^{2}$. Let $f_{3}(x, y, z)$, $f_{4}(x, y, z), f_{5}(x, y, z)$ be homogeneous polynomials of degrees $3,4,5$ respectively and let $B$ be a curve on $\mathbf{P}^{2}$ given by $f_{4}^{2}-4 f_{3} f_{5}=0$.

(1) If $B$ has no multiple components, no m-tuple points with $m \geqslant 4$ and no infinitely near $n$-tuple points with $n \geqslant 3$, then the double cover of $\mathbf{P}^{2}$ with branch locus $B$ is birational to a quintic surface of type III.

(2) If $B$ has no multiple components and exactly one infinitely near triple point, then the double cover of $\mathbf{P}^{2}$ with branch locus $B$ is birational to a quintic surface of type III-I', III-II ${ }_{\mathrm{a}},(\mathrm{I}, \mathrm{III})^{\prime}$ or $\left(\mathrm{II}_{\mathrm{a}}, \mathrm{III}\right)$.

Proof. Suppose $B$ satisfies either (1) or (2). The equation $f_{3}(x, y, z)+f_{4}(x, y, z)$ $+f_{5}(x, y, z)=0$ defines a quintic surface $S_{0}$ in $\mathbf{P}^{3}$ with a triple point $(0,0,0)$. Here $x, y, z$ are considered to be inhomogeneous coordinates. The projection from $(0,0,0)$ gives rise to a double cover over $\mathbf{P}^{2}$ with branch locus $B$. Hence the double cover of $\mathbf{P}^{2}$ with branch locus $B$ is birational to the quintic surface $S_{0}$. Let $X^{*}$ be the canonical resolution of the double cover. Then the formula (2.12) implies that

$$
\chi\left(X^{*}\right)= \begin{cases}4 & \text { if conditions in (1) are satisfied } \\ 3 & \text { if conditions in (2) are satisfied }\end{cases}
$$

Therefore, $S_{0}$ contains only one minimally elliptic triple point under the conditions in (1) and $S$ contains some minimally elliptic double point under the conditions in (2). 


\subsection{Families.}

THEOREM 9.4. $V_{3,2}$ is an irreducible quasivariety of dimension 48.

Proof. The irreducible group PGL(3) acts on $V_{3,2}=\mathrm{Hilb}\{\mathrm{III}\}$. To show that $V_{3,2}$ is an irreducible quasivariety it is enough to show that the set $W$ of all surfaces of type III with the triple point at some fixed point is an irreducible quasivariety. We may use affine coordinates and assume that the triple point is $(0,0,0)$. From Lemmas 9.2 and 9.3 we know that $W$ is the set of all irreducible polynomials $f_{3}(x, y, z)+f_{4}(x, y, z)+f_{5}(x, y, z)$ with the condition that the zero locus $B$ of $f_{4}^{2}-4 f_{3} f_{5}$ has no multiple components, no $m$-tuple points with $m \geqslant 4$ or infinitely near $n$-tuple point with $n \geqslant 3$. This is an open condition. Hence $W$ is an open subset of $\mathbf{P}^{N}$, where

$$
N=\left(\begin{array}{c}
3+2 \\
2
\end{array}\right)+\left(\begin{array}{c}
4+2 \\
2
\end{array}\right)+\left(\begin{array}{c}
5+2 \\
2
\end{array}\right)-1=45
$$

Therefore $V_{3,2}$ is irreducible and $\operatorname{dim} V_{3,2}=N+\operatorname{dim} P^{3}=48$.

Proposition 9.5. The set

$$
V_{2,1}^{\prime}=\operatorname{Hilb}\left\{\mathrm{III}_{-1}\right\} \cup \operatorname{Hilb}\left\{\mathrm{III}^{\prime} \mathrm{II}_{\mathrm{a}}\right\} \cup \operatorname{Hilb}\left\{\left(\mathrm{II}_{\mathrm{a}}, \mathrm{III}\right)\right\} \cup \operatorname{Hilb}\left\{(\mathrm{I}, \mathrm{III})^{\prime}\right\}
$$

is a family of quintic surfaces. The dimension of $V_{2,1}^{\prime}$ is 39 .

Proof. Let $W$ be the set of all members in $V_{2,1}^{\prime}$ with the triple point at a fixed point in $\mathbf{P}^{3}$. We want to show that $W$ is an irreducible quasivariety. Lemmas 9.2 and 9.3 imply that $W$ is the set of all irreducible polynomials $f_{3}(x, y, z)+f_{4}(x, y, z)+$ $f_{5}(x, y, z)$ with the condition that the zero locus $B$ of $f_{4}^{2}-4 f_{3} f_{5}$ has exactly one infinitely near triple point and no $m$-tuple points with $m \geqslant 4$, no other infinitely near $n$-tuple points with $n \geqslant 3$. Obviously this is an algebraic condition. Hence $W$ is a quasivariety.

Let $W^{\prime}$ be the subset of $W$ such that the zero locus of

$$
h(x, y)=f_{4}^{2}(x, y, 1)-4 f_{3}(x, y, 1) f_{5}(x, y, 1)
$$

has an infinitely near triple point at $(0,0)$, and such that $h(x, y)$ takes the form

$$
c_{1} x^{3}+\text { higher degree terms } \quad\left(c_{1} \neq 0\right)
$$

We write

$$
f_{3}(x, y, z)=\sum_{i+j+k=3} a_{i, j, k} x^{i} y^{j} z^{k}, \quad f_{4}(x, y, z)=\sum_{i+j+k=4} b_{i, j, k} x^{i} y^{j} z^{k}
$$

and

$$
f_{5}(x, y, z)=\sum_{i+j+k=5} c_{i, j, k} x^{i} y^{j} z^{k}
$$


Then $W^{\prime}$ is the set of all $f_{3}+f_{4}+f_{5}$ such that the coefficients of the 12 terms $1, x, y, x^{2}, x y, y^{2}, x^{2} y, x y^{2}, y^{3}, x y^{3}, y^{4}, y^{5}$ of $h(x, y)$ are zero. These 12 conditions are

$$
\begin{aligned}
& b_{0,0,4}^{2}-4 a_{0,0,3} c_{0,0,5}=0 \\
& 2 b_{0,0,4} b_{1,0,3}-4 a_{1,0,2} c_{0,0,5}-4 a_{0,0,3} c_{1,0,4}=0 \\
& 2 b_{0,0,4} b_{0,1,3}-4 a_{0,1,2} c_{0,0,5}-4 a_{0,0,3} a_{0,1,4}=0 \\
& 2 b_{0,0,4} b_{2,0,2}+b_{1,0,3}^{2}-4 a_{2,0,1} c_{0,0,5}-4 a_{1,0,2} c_{1,0,4}-4 a_{0,0,3} c_{2,0,3}=0 \\
& 2 b_{0,0,4} b_{1,1,2}+2 b_{0,1,3} b_{1,0,3}-4 a_{0,0,3} c_{1,1,3}-4 a_{0,1,2} c_{1,0,4} \\
& \quad-4 a_{1,0,2} c_{0,1,4}-4 a_{1,1,1} c_{0,0,5}=0 \\
& 2 b_{0,0,4} b_{0,2,2}+b_{0,1,3}^{2}-4 a_{0,0,3} c_{0,2,3}-4 a_{0,1,2} c_{0,1,4}-4 a_{0,2,1} c_{0,0,5}=0 \\
& 2 b_{0,0,4} b_{0,3,1}+2 b_{0,1,3} b_{0,2,2}-4 a_{0,0,3} c_{0,3,2}-4 a_{0,1,2} c_{0,2,3} \\
& \quad-4 a_{0,2,1} c_{0,1,4}-4 a_{0,3,0} c_{0,0,4}=0 \\
& 2 b_{0,0,4} b_{1,2,1}+2 b_{0,1,3} b_{1,1,2}+2 b_{1,0,3} b_{0,2,2}-4 a_{0,0,3} c_{1,2,2}-4 a_{0,1,2} c_{1,1,3} \\
& \quad-4 a_{1,0,2} c_{0,2,3}-4 a_{1,1,1} c_{0,1,4}-4 a_{1,2,0} c_{0,0,5}=0 \\
& 2 b_{0,0,4} b_{2,1,1}+2 b_{0,1,3} b_{1,1,2}+2 b_{1,0,3} b_{1,1,2}-4 a_{0,0,3} c_{2,1,2}-4 a_{0,1,2} c_{2,0,3} \\
& \quad-4 a_{1,0,2} c_{1,1,3}-4 a_{1,1,1} c_{1,0,4}-4 a_{2,1,0} c_{0,0,5}=0 \\
& 2 b_{0,0,4} b_{0,4,0}+2 b_{0,1,3} b_{0,3,1}+b_{0,2,2}^{2}-4 a_{0,0,3} c_{0,4,1}-4 a_{0,1,2} c_{0,3,2} \\
& \quad-4 a_{0,2,1} c_{0,2,3}-4 a_{0,3,0} c_{0,1,4}=0 \\
& 2 b_{0,0,4} b_{1,3,0}+2 b_{0,1,3} b_{1,2,1}+2 b_{1,0,3} b_{0,3,1}+2 b_{1,1,2} b_{0,2,2}-4 a_{0,0,3} c_{1,3,2} \\
& \quad-4 a_{0,1,2} c_{1,2,2}-4 a_{1,0,2} c_{0,3,2}-4 a_{1,1,1} c_{0,2,3}-4 a_{1,2,0} c_{0,1,4}=0 \\
& 2 b_{0,1,3} b_{0,4,0}+2 b_{0,2,2} b_{0,3,1}-4 a_{0,0,3} c_{0,5,0}-4 a_{0,1,2} c_{0,4,1} \\
& -4 a_{0,2,1} c_{0,3,2}-4 a_{0,3,0} c_{0,2,3}=0
\end{aligned}
$$

Let $\bar{W}$ be the set of all polynomials of the form $f_{3}+f_{4}+f_{5}$ satisfying the above 12 equations. It is easy to check that $\bar{W}$ is an irreducible variety of dimension 33 (= 45-12). Obviously $W^{\prime}$ is an open subvariety of $\bar{W}$.

The group GL(3) acts on $W$ freely. Each fibre of the morphism GL(3) $\times W^{\prime} \rightarrow W$ has dimension 6. Hence $W$ is an irreducible quasivariety of dimension 36 . Therefore $V_{2,1}^{\prime}$ is an irreducible quasivariety of dimension 39 .

REMARK 9.6. From Lemma 9.3 and Proposition 9.5, we can see that the III-I', III-II $_{\mathrm{a}}$ and (I, III) $)^{\prime}$ are specializations of $\left(\mathrm{II}_{\mathrm{a}}, \mathrm{III}\right)$.

10. Families of quintic surfaces of general type with elliptic double points. In this section we study the families of the remaining types of quintic surfaces of general type. According to Table 8.1, $V_{3,3}$ and $V_{3,4}$ are the simplest ones. Each of them contains only one type. In $\$ 10.1$ we will show that they are irreducible quasivarieties. Then we will discuss the other families in the subsequent subsections. Unfortunately we are not able to describe all families. So we leave this as an interesting open problem.

10.1 Families $V_{3,3}$ and $V_{3,4}$.

THEOREM 10.1. $V_{3,3}$ is an irreducible quasivariety of dimension 47. 
Proof. Since $V_{3,3}=\operatorname{Hilb}\{\mathrm{I}\}$, it suffices to show that Hilb $\{\mathrm{I}\}$ is an irreducible quasivariety of dimension 47 . Let $\bar{W}$ be the set of quintic surfaces given by

$$
c x^{2}+x f_{2}(x, y, z)+f_{4}(x, y, z)+f_{5}(x, y, z)=0,
$$

where $c \neq 0$ and $f_{i}(x, y, z)$ is a homogeneous polynomial of degree $i$. Then $\bar{W}$ is an open subset of $\mathbf{P}^{42}$. Obviously $W=\operatorname{Hilb}\{\mathrm{I}\} \cap \bar{W}$ is an open subset of $\bar{W}$. Since $\operatorname{Hilb}\{\mathrm{I}\}$ is the image of the morphism $\operatorname{PGL}(3) \times W \rightarrow$ Hilb $\{\mathrm{I}\}$ given by the group action, and any fibre of this map has dimension 10 , Hilb $\{\mathrm{I}\}$ is an irreducible quasivariety of dimension 47.

THEOREM 10.2. $V_{3.4}$ is an irreducible quasivariety of dimension 45.

Proof. Let $\bar{W}$ be the set of all quintic surfaces given by the equations

$$
c x^{2}+d y^{3}+x f_{2}(x, y, z)+f_{4}(x, y, z)+f_{5}(x, y, z)=0 \quad(c \neq 0)
$$

such that the double point $(0,0,0)$ is equivalent to the double point represented by $x^{2}+y^{3}+x z^{m}+z^{n}=0$ with $m \geqslant 4, n \geqslant 6$. Then $W$ is the set of all quintic surfaces given by the equations

$$
\begin{aligned}
\left(c_{1} x\right. & \left.+c_{2} y z+c_{3} z^{2}\right)^{2}+c_{4} y^{3}+y^{2} g_{2}(y, z)+y g_{4}(y, z) \\
& +\left(c_{1} x+c_{2} y z+c_{3} z^{2}\right)\left[y h_{1}(y, z)+h_{3}(y, z)\right]+x h_{4}(y, z) \\
& +x^{2}\left[m_{1}(x, y)+m_{2}(x, y, z)+m_{3}(x, y, z)\right] \\
& +c_{5}\left(c_{1} x+c_{2} y z+c_{3} z^{2}\right)^{2} z=0
\end{aligned}
$$

where $g_{i}, h_{i}, m_{i}$ are homogeneous polynomials of degree $i$. Hence $W=\bar{W}$ $\cap \mathrm{Hilb}\left\{\mathrm{II}_{\mathrm{a}}\right\}$ is an irreducible quasivariety of dimension 41. A fibre of the groupaction map PGL(3) $\times W \rightarrow \operatorname{Hilb}\left\{\mathrm{II}_{\mathrm{a}}\right\}$ has dimension 11 . Therefore $\mathrm{Hilb}\left\{\mathrm{II}_{\mathrm{a}}\right\}=V_{3,4}$ is an irreducible quasivariety of dimension 45.

Notice that in the proofs of Theorems 10.1 and 10.2, we inspect the quintic equations directly. The same method is not effective for other types of surfaces. As the double points become worse, the conditions on the quintic equations will be very complicated. So we try to use the triple covering map induced by the projection from one double point.

10.2 Representations as triple covers. Let $S_{0}$ be a normal quintic surface with a double point $p$. Let $\sigma: S^{\prime} \rightarrow S_{0}$ be the blowing-up of $S_{0}$ at $p$. Let $\varphi^{\prime}: S^{\prime} \rightarrow \mathbf{P}^{2}$ be the morphism induced by the projection from the point $p$. Let $\tau: S^{\#} \rightarrow S^{\prime}$ be the minimal resolution of $S^{\prime}$. Then $\varphi=\varphi^{\prime} \circ \tau$ is a morphism from $S^{\#}$ to $\mathbf{P}^{2}$, which is generically finite of degree 3 . Unlike the triple point case, the surface $S^{\#}$ does not need to be a minimal resolution of $S_{0}$. Let $\tilde{S}$ be a minimal model of $S^{\#}$.

Similar to the cases discussed in $§ 9.1$, all quintic surfaces of general type with only elliptic double points as essential singularities can be described by the branch locus of the morphism $\varphi$ in some way. For the simplicity of arguments, we only consider a generic member from each type of quintic surfaces in Table 8.1. Since we discuss the general properties (irreducibility, dimension, etc.) of the families, this restriction does not affect the results. 
Let $S_{0}$ be a quintic surface of type I. Since we are considering the generic cases, we may assume that the fundamental cycle $Z$ is a nonsingular elliptic curve with $Z^{2}=-2$. In this case $S^{\#}=\tilde{S}, p_{g}(\tilde{S})=3, q(\tilde{S})=0, K_{\tilde{S}}^{2}=3$.

Since $\left|K_{\tilde{S}}\right|$ is cut out by all hyperplanes of $\mathbf{P}^{3}$ passing through the point $p,\left|K_{\tilde{S}}\right|$ has neither fixed components nor base points. Hence the linear system $\left|K_{\tilde{S}}\right|$ defines a triple covering map $\varphi: \tilde{S} \rightarrow \mathbf{P}^{2}$ which is induced by the projection from $p$.

Denote the line bundle $\operatorname{Spec}_{\mathcal{O}_{\mathbf{P} 2}}\left(\operatorname{Symm} \mathcal{O}_{\mathbf{P}^{2}}(n)\right)$ of degree $n$ on $\mathbf{P}^{2}$ by $F_{n}$.

THEOREM 10.3. Let $\varphi$ be the triple covering map from $\tilde{S}$ onto $\mathbf{P}^{2}$ as defined above. Then there is a morphism $i: \tilde{S} \rightarrow F_{2}$, which is a birational morphism onto a surface without essential singularities, such that $\varphi=\operatorname{pr} \circ i$, where $\mathrm{pr}: F_{2} \rightarrow \mathbf{P}^{2}$ is the projection of the line bundle $F_{2}$. Moreover, $\varphi$ induces a double cover of $Z$ over a line in $\mathbf{P}^{2}$.

Conversely, let $\psi$ be the fibre coordinate of the line bundle $F_{2}$, let $q$ and $r$ be homogeneous polynomials in $z_{0}, z_{1}, z_{2}$ of degrees 4 and 6 respectively. If the surface $S$ in $F_{2}$ defined by $\psi^{3}+q \psi+r=0$ satisfies the conditions that

(i) $S$ has no essential singularities;

(ii) there is a line $L$ on $\mathbf{P}^{2}$ such that $\mathrm{pr}^{-1}(L)$ on $S$ is the union of a rational curve and a nonsingular elliptic curve,

then $S$ is birational to a normal quintic surface with a minimally elliptic double point of type I.

Proof. Let $\tilde{S}$ be the minimal resolution of a normal quintic surface $S_{0}$ with a minimally elliptic double point $p$ of type I. Let $\left\{\varphi_{0}, \varphi_{1}, \varphi_{2}\right\}$ be a basis of $\left|K_{\tilde{S}}\right|$. By Theorem 2.3, the linear system $\left|2 K_{\tilde{S}}\right|$ defines a morphism $\varphi_{\left|2 K_{\tilde{s}}\right|}$ into $\mathbf{P}^{6}$. Horikawa [8, II] showed that $\varphi_{\left|2 K_{\tilde{S}}\right|}$ is a birational morphism onto its image for any minimal surface $\tilde{S}$ with $p_{g}(\tilde{S})=3, q(\tilde{S})=0, K_{\tilde{S}}^{2}=3$. Since the plurigenus $P_{2}=7$, there exists $\psi \in H^{0}\left(\tilde{S}, \mathcal{O}\left(2 K_{\tilde{S}}\right)\right)$ such that $\varphi_{i} \varphi_{j}(0 \leqslant i \leqslant j \leqslant 2)$ and $\psi$ form a basis of $H^{0}\left(\tilde{S}, \mathcal{O}\left(2 K_{\tilde{S}}\right)\right)$. The 49 products

$$
\varphi_{i_{1}} \cdots \varphi_{i_{6}}, \quad \psi \varphi_{i_{1}} \cdots \varphi_{i_{4}}, \quad \psi^{2} \varphi_{i_{1}} \varphi_{i_{2}}
$$

are linearly independent in $H^{0}\left(\tilde{S}, \mathcal{O}\left(6 K_{\tilde{S}}\right)\right)$ because $\varphi_{\left|2 K_{\tilde{S}}\right|}$ is birational. By Theorem 2.3, $P_{6}=49$. Hence $\psi^{3}$ is a linear combination of the terms in (10.1). In other words, $\psi$ satisfies $\psi^{3}+A \psi^{2}+q \psi+r=0$, where $A, q, r$ are homogeneous polynomials in $\varphi_{0}, \varphi_{1}, \varphi_{2}$ of degrees $2,4,6$ respectively. A linear transform $\psi^{\prime}=\psi+A / 3$ can kill the $\psi^{2}$ term. Hence we may assume that $\psi$ satisfies

$$
\psi^{3}+q \psi+r=0
$$

Regard $\varphi_{0}, \varphi_{1}, \varphi_{2}$ as homogeneous coordinates of $\mathbf{P}^{2}$ and $\psi$ as fibre coordinate of the line bundle $F_{2}$. Then (10.2) defines a surface $S \subset F_{2}$. We can see that $S$ is isomorphic to the image of $\varphi_{\left|2 K_{\tilde{s}}\right|}$ in $\mathbf{P}^{6}$, which has no essential singularities [5]. Actually the map from $S$ to $\mathbf{P}^{6}$ is given by

$$
\left(\varphi_{0}, \varphi_{1}, \varphi_{2}, \psi\right) \mapsto\left(\varphi_{0}^{2}, \varphi_{0} \varphi_{1}, \varphi_{0} \varphi_{2}, \varphi_{1}^{2}, \varphi_{1} \varphi_{2}, \varphi_{2}^{2}, \psi\right)
$$


Now the following diagram commutes:

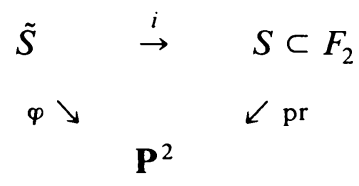

where $i$ is induced by $\varphi_{\left|2 K_{\tilde{S}}\right|}$.

As $K_{\tilde{S}} Z=2, \varphi_{*}(Z)$ is a degree two curve. Hence $\varphi(Z)$ is either a conic or a line. By the exact sequence

$$
0 \rightarrow \mathcal{O}_{\tilde{S}}\left(K_{\tilde{S}}-Z\right) \rightarrow \mathcal{O}_{\tilde{S}}\left(K_{\tilde{S}}\right) \rightarrow \mathcal{O}_{Z}(2) \rightarrow 0
$$

we infer that $\operatorname{dim} H^{0}\left(\tilde{S}, \mathcal{O}_{\tilde{S}}\left(K_{\tilde{S}}-Z\right)\right) \geqslant 1$. So there is some $\varphi \in\left|K_{\tilde{S}}\right|$ such that $\varphi$ vanishes on $Z$. That means that $\varphi(Z)$ is a line. Hence $\varphi: Z \rightarrow \varphi(Z)$ is a double cover. This finishes the proof of the first part of the theorem.

Conversely, suppose there are $\psi, q, r$ satisfying conditions (i) and (ii). Let $S$ be the surface in $F_{2}$ defined by equation (10.2). Without loss of generality, we may assume that $S$ is nonsingular. Let $Z$ be a nonsingular elliptic curve on $S$ as implied by condition (ii). Let $\varphi^{*}(L)=Z+M$. Since the branch locus $B$ of the triple covering map is given by the equation $4 q^{3}+27 r^{2}=0, B$ has degree 12 . Since $\varphi$ induces a double covering map from the nonsingular elliptic curve onto a line, $L$ meets $B$ at four distinct points transversally (these four points are the images of the ramification points of $\varphi: Z \rightarrow L)$ and $(L, B)_{a} \equiv 0(\bmod 2)$ for any other point $a$. Hence $Z M=4$. So $2=Z(Z+M)=Z^{2}+Z M=Z^{2}+2$ which implies $Z^{2}=-2$. Thus $\left(\varphi^{*}(L)+Z\right)^{2}=5$.

Since pr: $S \rightarrow \mathbf{P}^{2}$ is an affine morphism, $H^{i}(S, \mathscr{F}) \cong H^{i}\left(\mathbf{P}^{2}, \varphi_{*} \mathscr{F}\right)$ for all $i$ and for any coherent sheaf $\mathscr{F}$ on $S$. On the other hand, $\varphi_{*} \mathcal{O}_{S} \cong \mathcal{O}_{\mathbf{P}^{2}} \oplus \mathcal{O}_{\mathbf{P}^{2}}(-2) \oplus$ $\mathcal{O}_{\mathbf{P}^{2}}(-4)$. Hence

$$
H^{i}\left(S, \mathcal{O}_{S}\left(\varphi^{*}(L)\right)\right) \cong H^{i}\left(\mathbf{P}^{2}, \mathcal{O}_{\mathbf{P}^{2}}(1) \oplus \mathcal{O}_{\mathbf{P}^{2}}(-1) \oplus \mathcal{O}_{\mathbf{P}^{2}}(-3)\right) \quad \text { for } i=0,1,2 .
$$

Thus

$$
\operatorname{dim} H^{i}\left(S, \mathcal{O}_{S}\left(\varphi^{*}(L)\right)\right)= \begin{cases}3, & i=0 \\ 0, & i=1 \\ 1, & i=2\end{cases}
$$

The exact sequence

$$
0 \rightarrow \mathcal{O}_{S}\left(\varphi^{*} L\right) \rightarrow \mathcal{O}_{S}\left(\varphi^{*} L+Z\right) \rightarrow \mathcal{O}_{Z} \rightarrow 0
$$

implies that $\operatorname{dim} H^{0}\left(S, \mathcal{O}_{S}\left(\varphi^{*} L+Z\right)\right)=4$.

Obviously, $\left|\varphi^{*} L+Z\right|$ has no fixed points; it defines a morphism from $S$ into $\mathbf{P}^{3}$. Since $\left(\varphi^{*} L+Z\right)^{2}=5$, this morphism must be birational onto a quintic surface in $\mathbf{P}^{3}$. Since $\left(\varphi^{*} L+Z\right) Z=0$, the curve $Z$ contracts to a point under this morphism. Since $K_{S} D \geqslant 0$ for any effective divisor $D$ on $S, S$ is a minimal surface with $p_{g}(S)=3, q(S)=0, K_{S}^{2}=3$. Therefore the birational image of $S$ in $\mathbf{P}^{3}$ must be a quintic surface with a minimally elliptic double point of type $I$.

REMARK. Actually the image of $i$ is the normalization of $S^{\prime}$, where $S^{\prime}$ is the blowing-up of $S_{0}$ at $p$. 
The next lemma gives the conditions on $q\left(z_{0}, z_{1}, z_{2}\right)$ and $r\left(z_{0}, z_{1}, z_{2}\right)$ for (ii) in Theorem 10.3.

LEMMA 10.4. Condition (ii) is true if and only if there is a line $L: \lambda\left(z_{0}, z_{1}, z_{2}\right)=0$ on $\mathbf{P}^{2}$ and homogeneous polynomials $\alpha, \beta, h_{1}, h_{2}$ in $z_{0}, z_{1}, z_{2}$ of degrees $2,4,3,5$ respectively such that

(i) $q=\lambda h_{1}+\beta-\alpha^{2}, r=\lambda h_{2}-\alpha \beta$;

(ii) the zero locus of $\alpha^{2}-4 \beta$ meets $L$ at four distinct points transversally.

Proof. The inverse image $\mathrm{pr}^{-1}(L)$ of $L$ splits if and only if

$$
\psi^{3}+q \psi+r \equiv\left(\psi^{2}+\alpha \psi+\beta\right)(\psi-\alpha)(\bmod \lambda)
$$

which is exactly condition (i). The equation $\psi^{2}+\alpha \psi+\beta=0$ defines a double cover over $L$. This curve is a nonsingular elliptic curve if and only if the four branch points are distinct. This is exactly condition (ii).

REMARK. The above triple covering map can be directly derived from the original quintic equation

$$
f_{2}(x, y, z)+f_{3}(x, y, z)+f_{4}(x, y, z)+f_{5}(x, y, z)=0 \text {. }
$$

Since $p$ is not a rational double point, we may assume that $f_{2}(x, y, z)=\lambda(x, y, z)^{2}$ where $\lambda(x, y, z)$ is a linear form. The surface $S^{\prime}$ is given by the equation

$$
t^{3} f_{5}\left(z_{0}, z_{1}, z_{2}\right)+t^{2} f_{4}\left(z_{0}, z_{1}, z_{2}\right)+t f_{3}\left(z_{0}, z_{1}, z_{2}\right)+f_{2}\left(z_{0}, z_{1}, z_{2}\right)=0
$$

where $z_{0}, z_{1}, z_{2}$ are regarded as the homogeneous coordinates of $\mathbf{P}^{2}$. Note that $\lambda\left(z_{0}, z_{1}, z_{2}\right) \mid f_{3}\left(z_{0}, z_{1}, z_{2}\right)$ because $p$ is not a rational double point. Hence $S^{\prime}$ is singular along the curve $t=0, \lambda\left(z_{0}, z_{1}, z_{2}\right)=0$. Normalizing $S^{\prime}$, we get the surface $\tilde{S}$, which is given by

$$
\psi^{3}+g_{2}\left(z_{0}, z_{1}, z_{2}\right) \psi^{2}+f_{4}\left(z_{0}, z_{1}, z_{2}\right) \psi+\lambda\left(z_{0}, z_{2}, z_{2}\right) f_{5}\left(z_{0}, z_{1}, z_{2}\right)=0
$$

where

$$
g_{2}\left(z_{0}, z_{1}, z_{2}\right)=f_{3}\left(z_{0}, z_{1}, z_{2}\right) / \lambda\left(z_{0}, z_{1}, z_{2}\right) .
$$

Substitute $\psi$ by $\psi-g_{2}\left(z_{0}, z_{1}, z_{2}\right) / 3$. Then (10.3) becomes

$$
\psi^{3}+\left(f_{4}-g_{2}^{2} / 3\right) \psi+\left(\lambda f_{5}+2 g_{2}^{3} / 27-g_{2} f_{4} / 3\right)=0
$$

This is equation (10.2).

Note that $q$ and $r$ in (10.2) are expressed as polynomials of the coefficients of the original quintic surface; an alternative proof of Theorem 10.1 can be written down immediately by using Theorem 10.3.

10.3 The family Hilb $\{(\mathrm{I}, \mathrm{I})\} \cup \operatorname{Hilb}\left\{\mathrm{I}^{2,0}\right\}$. Suppose that $S_{0}$ is a generic member of $\operatorname{Hilb}\{(I, I)\}$ with two elliptic double points $p_{1}, p_{2} \in S_{0}$. Then the projection map from $p_{1}$ gives rise to a triple covering map, which is also locally defined by (10.4). Since the line $L_{0}$ passing through $p_{1}$ and $p_{2}$ does not lie on $S_{0}$, the image of $p_{2}$ on $\mathbf{P}^{2}$ is not on the line $\lambda=0$. Since $S_{0}$ is generic, we may assume that the triple covering map is not totally ramified at $p_{2}$. Thus the branch locus has a quadruple point at the image of $p_{2}$. 
Now suppose that $S_{0}$ is a surface with a double point $p$ of type $\mathrm{I}^{2,0}$. Then the projection map from $p$ gives rise to a triple covering map. If $S_{0}$ is a generic member, then it is easy to see that the branch locus has a quadruple point on the line $\lambda=0$. Therefore surfaces of type $(I, I)$ and $I^{2,0}$ are in the same family. In fact, $I^{2,0}$ is the specialization of $(I, I)$. Let $V_{2,1}^{\prime \prime}$ be the closure of $\operatorname{Hilb}\{(I, I)\} \cup \operatorname{Hilb}\left\{I^{2,0}\right\}$ in $V_{2,1}$.

THEOREM 10.5. The sets $V_{2,1}^{\prime}$ and $V_{2,1}^{\prime \prime}$ are irreducible components of $V_{2,1}$ with $\operatorname{dim} V_{2,1}^{\prime}=V_{2,1}^{\prime \prime}=39$.

Proof. From (9.5) we see that $V_{2,1}^{\prime}$ is an irreducible quasivariety with dimension 39. Now we show that $V_{2,1}^{\prime \prime}$ has the same property. Since the method is essentially the same as that in $\S \S 10.1$ and 10.2 , we only sketch the proof.

Let $W$ be the set of all members in $\operatorname{Hilb}\{(I, I)\} \cup \operatorname{Hilb}\left\{I^{2,0}\right\}$ whose equations are

$$
f(x, y, z)=\lambda(x, y, z)^{2}+f_{3}(x, y, z)+f_{4}(x, y, z)+f_{5}(x, y, z)=0
$$

where $\lambda(x, y, z)$ is a linear form.

Let $W^{\prime}$ be the subset of $W$ consisting of all members such that the branch locus of the above-mentioned triple covering has a quadruple point at $x=0, y=0$. This imposes ten conditions on the coefficients of $f(x, y, z)$. We can write all these conditions by using equation (10.4), but we prefer not to because they will be quite messy. Anyhow, $W^{\prime}$ is an irreducible quasivariety. Hence $W$ is also irreducible. Furthermore, $\operatorname{dim} V_{2,1}^{\prime \prime}=\operatorname{dim} V_{3,3}-8=39$.

Recall that $V_{2,1}=V_{2,1}^{\prime} \cup V_{2,1}^{\prime \prime}$, where $V_{2,1}^{\prime}$ is relatively closed in $V_{2,1}$ because quintic surfaces with triple points cannot deform into a quintic surface with only double points. Since $\operatorname{dim} V_{2,1}^{\prime \prime}=\operatorname{dim} V_{2,1}^{\prime}$, either they are equal or they are two irreducible components of $V_{2,1}$. Obviously only the latter case can happen.

REMARK. It is an interesting problem to find out what $V_{2,1}^{\prime} \cap V_{2,1}^{\prime \prime}$ looks like.

\section{REFERENCES}

1. M. Artin, On Enriques' surfaces, Thesis, Harvard Univ., Cambridge, Mass., 1960.

2. __ On isolated rational singularities of surfaces, Amer. J. Math. 88 (1966), 129-136.

3. Some numerical criteria for contractibility of curves on an algebraic surface, Amer. J. Math. 84 (1962), 485-496.

4. A. Beauville, Surfaces algébriques complexes, Asterisque 54 (1978), 1-172.

5. E. Bombieri, Canonical models of surfaces of general type, Inst. Hautes Étude Sci. Publ. Math. 42 (1973), 171-219.

6. L. Brenton, On singular complex surfaces with vanishing geometric genus, and pararational singularities, Compositio Math. 43 (1981), 297-315.

7. P. Griffiths and J. Harris, Principles of algebraic geometry, Wiley, New York, 1978.

8. E. Horikawa, Algebraic surfaces of general type with small $c_{1}^{2}$. I, II, Ann. of Math. (2) 104 (1976), 357-387; Invent. Math. 37 (1976), 121-155.

9. On deformations of quintic surfaces, Invent. Math. 31 (1975), 43-85.

10. D. Kirby, The structure of an isolated multiple point of surface. I, II, Proc. London Math. Soc. 6 (1956), 597-609; 7 (1957), 1-28.

11. K. Kodaira, Pluricanonical systems on algebraic surfaces of general type, J. Math. Soc. Japan 20 (1968), 170-192.

12. H. Laufer, On minimally elliptic singularities, Amer. J. Math. 99 (1977), 1257-1295.

13. D. Mumford, The topology of normal singularities of an algebraic surface and a criterion for simplicity, Inst. Hautes Étude Sci. Publ. Math. 9 (1961), 5-22. 
14. R. Miranda, Triple covers in algebraic geometry, preprint.

15. M. Reed, Elliptic Gorenstein singularities of surfaces, preprint.

16. J. Rotman, An introduction to homological algebra, Academic Press, New York, 1979.

17. R. Hartshorne, Algebraic geometry, Springer-Verlag, New York, Heidelberg and Berlin, 1977.

18. S. S-T. Yau, Hypersurface weighted dual graphs of normal singularities of surfaces, Amer. J. Math. 101 (1979), 761-812.

19. On maximally elliptic singularities, Trans. Amer. Math. Soc. 257 (1980), 269-329.

20. Jin-Gen Yang, On quintic surfaces of general type, Thesis, MIT, Cambridge, Mass., 1984.

21. J. Mather and S. S-T. Yau, Criterion for biholomorphic equivalence of isolated hypersurface singularities, Proc. Nat. Acad. Sci. U.S.A. 78 (1981), 5946-5947.

Department of Mathematics, University of TeXas, Austin, TeXas 78712 